\title{
Translation initiation factors are not required for Dicistroviridae IRES function in vivo
}

\author{
NILSA DENIZ, ${ }^{1,2}$ ERIK M. LENARCIC, ${ }^{1}$ DORI M. LANDRY, and SUNNIE R. THOMPSON \\ Department of Microbiology, University of Alabama at Birmingham, Birmingham, Alabama 35294, USA
}

\begin{abstract}
The cricket paralysis virus (CrPV) intergenic region (IGR) internal ribosome entry site (IRES) uses an unusual mechanism of initiating translation, whereby the IRES occupies the P-site of the ribosome and the initiating tRNA enters the A-site. In vitro experiments have demonstrated that the CrPV IGR IRES is able to bind purified ribosomes and form 80S complexes capable of synthesizing small peptides in the absence of any translation initiation factors. These results suggest that initiation by this IRES is factor-independent. To determine whether the IGR IRES functions in the absence of initiation factors in vivo, we assayed IGR IRES activity in various yeast strains harboring mutations in canonical translation initiation factors. We used a dicistronic reporter assay in yeast to determine whether the CrPV IGR IRES is able to promote translation sufficient to support growth in the presence of various deletions or mutations in translation initiation factors. Using this assay, we have previously shown that the CrPV IGR IRES functions efficiently in yeast when ternary complexes (eIF2•GTP•initiator tRNA ${ }^{\text {met }}$ ) are reduced. Here, we demonstrate that the CrPV IGR IRES activity does not require the eukaryotic initiation factors elF4G1 or elF5B, and it is enhanced when eIF2B, the eIF3b subunit of eIF3, or eIF4E are impaired. Taken together, these data support a model in which the CrPV IGR IRES is capable of initiating protein synthesis in the absence of any initiation factors in vivo, and suggests that the CrPV IGR IRES initiates translation by directly recruiting the ribosomal subunits in vivo.
\end{abstract}

Keywords: internal ribosome entry site; IGR IRES; cricket paralysis virus; translation initiation; $S$. cerevisiae

\section{INTRODUCTION}

Eukaryotic protein synthesis can be initiated by two mechanisms: cap-dependent and cap-independent. The vast majority of mRNAs initiate translation through the cap-dependent mechanism, requiring a $5^{\prime}{ }^{77} \mathrm{GpppN}$ cap structure recognized by the eIF4F complex, which is composed of the cap-binding protein (eIF4E), scaffolding protein (eIF4G), and helicase (eIF4A). The 40S ribosomal subunit is recruited to the $5^{\prime}$ end as a $43 \mathrm{~S}$ preinitiation complex (PIC) consisting of the $40 \mathrm{~S}$ ribosomal subunit plus the multifactor complex (MFC: eIF1, eIF1A, eIF3, eIF5, and the ternary complex [eIF2, GTP, and Met-tRNA $\left.\left.\mathrm{i}_{\mathrm{Met}}\right]\right)$. The PIC scans down the mRNA until it reaches the start codon.

\footnotetext{
${ }^{1}$ These authors contributed equally to this work.

${ }^{2}$ Present address: Histology, Drug Development Division, Southern Research Institute, Birmingham, AL 35205, USA.

Reprint requests to: Sunnie R. Thompson, Department of Microbiology, 1530 3rd Avenue South, BBRB 466/21, University of Alabama at Birmingham, Birmingham AL 35294, USA; e-mail: sunnie@uab.edu; fax: (205) 934-9256.

Article published online ahead of print. Article and publication date are at http://www.rnajournal.org/cgi/doi/10.1261/rna.1315109.
}

At that point, Met-tRNA $\mathrm{A}_{\mathrm{i}}^{\mathrm{Met}}$ base-pairs with the AUG, eIF5 stimulates eIF2-GTP hydrolysis, eIF2-GDP is released along with other factors, eIF5B-GTP is recruited to stimulate $60 \mathrm{~S}$ ribosomal subunit joining, and eIF1A and eIF5B-GDP are released to generate the translationally active $80 \mathrm{~S}$ complex (Fringer et al. 2007; Hinnebusch et al. 2007). After each round of initiation, eIF2-GDP must be recycled by eIF2B (a guanine nucleotide exchange factor) for a new round of initiation. Regulation of eIF2 recycling is an important translational control mechanism. During cell stress the $\alpha$ subunit of eIF2 is phosphorylated, which prevents recycling and results in a dramatic decrease in global translation.

The cap-independent translation initiation mechanism requires an internal ribosome entry site (IRES) to recruit ribosomes internally, thus bypassing the need for a 5 ' capstructure. Following their discovery in picornaviral RNAs (Jang et al. 1988; Pelletier and Sonenberg 1988), IRESs have also been identified in cellular messages that encode proteins involved in growth, apoptosis, differentiation, tumorigenesis, and hypoxia (Jackson 2005). Furthermore, members of the Retroviridae and Herpesviridae families of viruses have been shown to express certain proteins from IRESs (Berlioz and Darlix 1995; Vagner et al. 1995; Bieleski 
and Talbot 2001; Buck et al. 2001; Grundhoff and Ganem 2001; Low et al. 2001; Brasey et al. 2003; Herbreteau et al. 2005; Nicholson et al. 2006). Several reports have described IRES activity in vivo in yeast using a sensitive reporter assay or growth assay (Paz et al. 1999; Zhou et al. 2001; Thompson et al. 2001; Komar et al. 2003; Gilbert et al. 2007).

Very little is known about the mechanism of ribosomal recruitment by IRESs. Some IRESs, such as picornaviral IRESs, require a subset of the translation initiation factors. For example, poliovirus has been shown to require eIF2, eIF3, eIF4A, and the $\mathrm{C}$ terminus of eIF4G for in vitro activity (Jackson 2005). In contrast, IGR IRESs are capable of binding purified $40 \mathrm{~S}$ and $60 \mathrm{~S}$ ribosomal subunits to form $80 \mathrm{~S}$ complexes in the absence of any initiation factors (Hellen and Sarnow 2001). The IGR IRES forms a compact structure with three pseudoknot (PK) structures. PK2 and PK3 make a compact core that is capable of binding to $40 \mathrm{~S}$ ribosomal subunits in vitro, while PK1 is positioned into the P-site of the ribosome (Wilson et al. 2000a; Kanamori and Nakashima 2001; Spahn et al. 2004; Costantino and Kieft 2005; Schuler et al. 2006; Costantino et al. 2008). The mechanisms of the hepatitis C virus (HCV) and CrPV IGR IRESs represent the simplest and best studied IRESs to date. Interestingly, although they are very different in sequence and structure (Spahn et al. 2001, 2004; Boehringer et al. 2005; Pfingsten et al. 2006; Schuler et al. 2006; Costantino et al. 2008; Pfingsten and Kieft 2008), they share some similarities in their mechanism for binding ribosomes. In vitro they can both bind directly to $40 \mathrm{~S}$ subunits, occupy the E-site of the ribosome, result in similar conformational changes of the 40S subunit prior to 60S joining (Pfingsten and Kieft 2008), and initiate protein synthesis in the absence of any initiation factors (Jan et al. 2003; Pestova and Hellen 2003; Lancaster et al. 2006). However, another report found that at a minimum the HCV IRES requires additional initiation factors, eIF3 and eIF5B, to initiate protein synthesis in vitro (Terenin et al. 2008). Thus, the question remains whether initiation-factor-independent initiation is physiologically relevant. In vitro biochemicalassays have suggested that components of the ternary complex or the MFC prevent IGR IRES complex formation with ribosomes. The addition of eIF5B to a preformed 40S, eIF1, eIF1A, and eIF3 complex enhances IGR IRES 80S complex formation (Pestova et al. 2004). In contrast, addition of excess eIF5B to an in vitro translation assay prevents pseudotranslocation, the movement of the ribosome by one codon in the absence of peptide bond formation (Wilson et al. 2000a).

Very little is known about the requirements of the CrPV IGR IRES in vivo aside from the observation that $\mathrm{CrPV}$ IGR IRES activity is enhanced when ternary complexes are low (Thompson et al. 2001). In this study, we have used yeast genetics to elucidate the molecular mechanisms of the CrPV IGR IRES in vivo to establish the physiological relevance of the model for CrPV IGR IRES-mediated translation initiation. We report that the IGR IRES does not require ternary complex, MFC formation, cap-binding protein, eIF4G1, or eIF5B in vivo. Taken together, these data support a model in which the IGR IRES initiates translation in vivo without the canonical initiation factors. Similar to cellular IRES activity, CrPV IGR IRES activity is enhanced in cells when cap-dependent translation is decreased (Cornelis et al. 2000; Pyronnet et al. 2000; Fernandez et al. 2001; Stoneley and Willis 2004).

\section{RESULTS}

\section{The CrPV IGR IRES functions efficiently in yeast cells when eIF2B is mutated}

Growth assays were used to assess initiation factor requirements for the CrPV IGR IRES in vivo. We assayed IRES activity by placing expression of the URA3 open reading frame under the control of the IGR IRES in a dicistronic reporter in which the tightly regulated CUP1 promoter (Butt et al. 1984; Mascorro-Gallardo et al. 1996) was used to drive transcription of a dicistronic LEU2 IGR URA3 reporter (Fig. 1A). The first cistron, LEU2, is translated by the cap-dependent mechanism of initiation, while the downstream cistron, URA3, is translated by a cap-independent mechanism by the IGR IRES. To determine whether a given initiation factor is required for IRES-mediated translation in vivo, yeast strains harboring mutations in that initiation factor were transformed with the dicistronic reporter and assayed for growth by serial dilutions on media that lacked uracil. If the initiation factor is not required for IRES function, then we expect to see an enhancement of IGR IRES activity and thus an increase in cell growth on the selective media. However, if this is not the case, then we expect to see no growth on selective media similar to the wild-type IGR IRES in wild-type cells.

We have shown previously that the IGR IRES functions efficiently when the abundance of ternary complexes was lowered (Thompson et al. 2001). We extended these studies by examining the IGR IRES function in yeast cells harboring mutations in eIF2B, the guanine nucleotide exchange factor for eIF2. We examined IGR IRES activity in two different yeast strains, which harbor mutations in either the catalytic (GCD6) or regulatory (GCD7) subunit of eIF2B (Bushman et al. 1993). Mutations in subunits of the guanine nucleotide exchange factor decrease the amount of eIF2-GTP that can be recycled from eIF2-GDP to form ternary complexes. $g c d 6-1$ and $g c d 7-201$ along with their respective isogenic wild-type strains were transformed with the dicistronic reporter plasmid. To adjust for the intrinsic growth rate differences between the wild-type and mutant yeast strains observed on synthetic complete (SC) media (Fig. 1B) the strains were allowed to grow until the plate with the uracil (Fig. 1C; right, +uracil) was fully grown, resulting in different growth times for the wild-type and the 
A

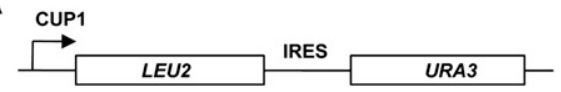

B

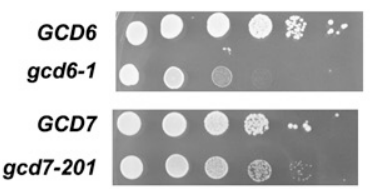

\section{C}

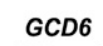

(4 Days)
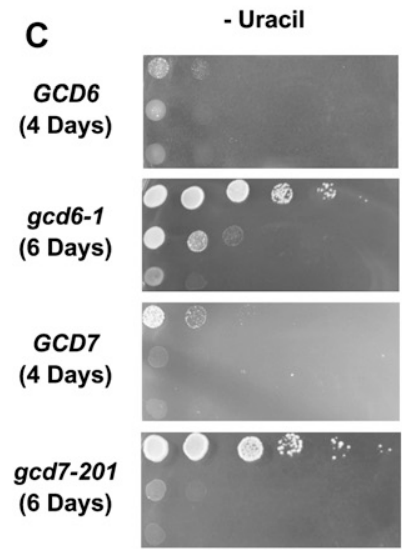

gcd7-201 (6 Days)
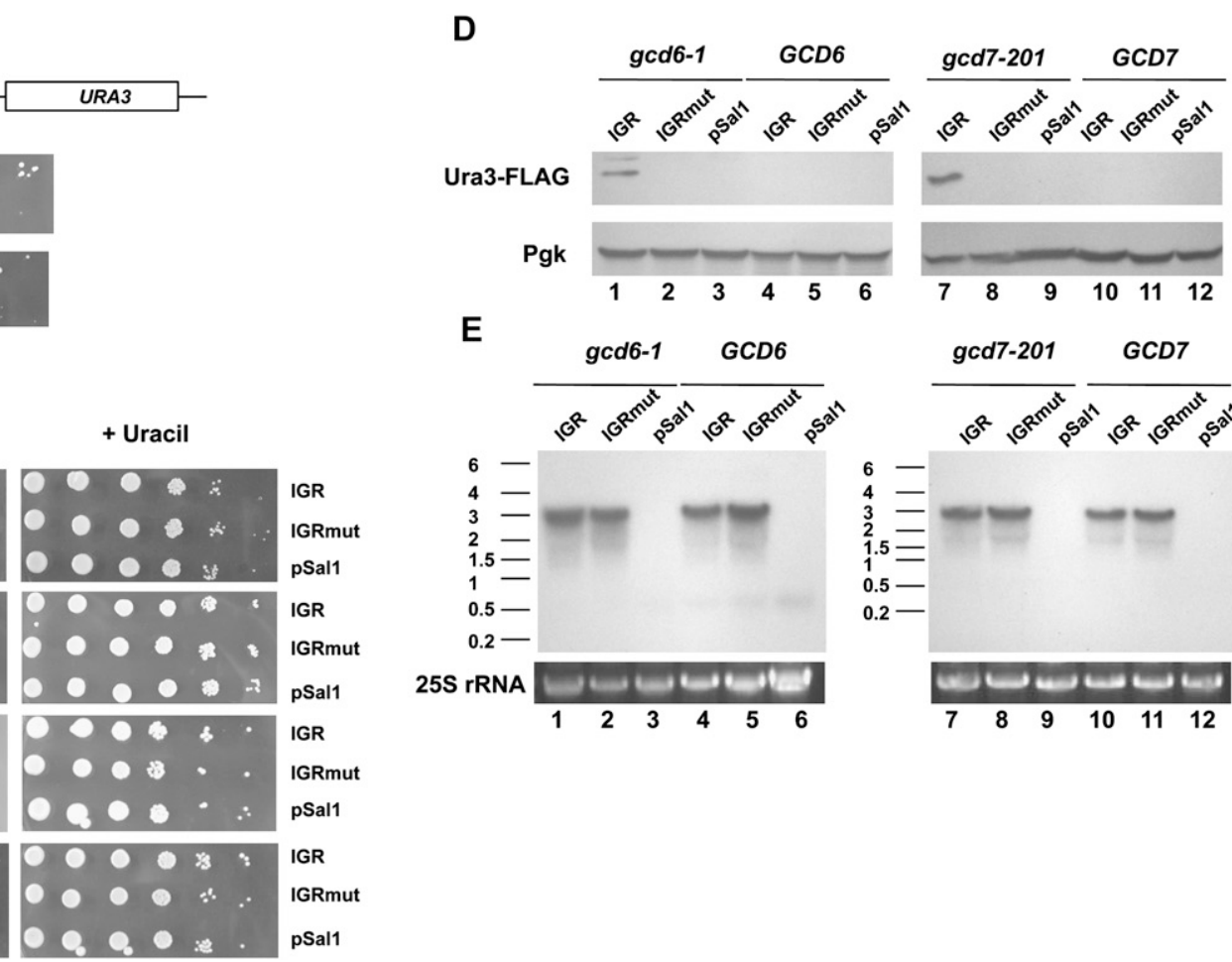

E
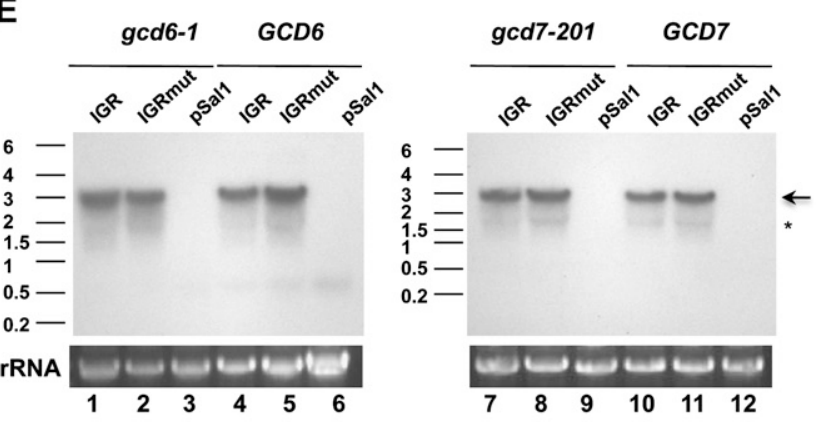

FIGURE 1. CrPV IGR IRES functions efficiently when the catalytic or regulatory subunits of eIF2B are mutated. (A) Diagram of the LEU2 IGR URA3 dicistronic reporter; the CUP1 promoter that drives transcription of the reporter is indicated by an arrow. (B) Serial dilutions of wild-type (GCD7 or GCD6) and mutant ( $g c d 7-201$ or $g c d 6-1)$ eIF2B yeast strains on SD complete media demonstrate relative growth phenotypes. (C) Growth assays on SD media with $100 \mu \mathrm{M} \mathrm{CuSO}_{4}$, supplemented without (left) or with (right) uracil. $(D)$ Western analysis of Flag-tagged Ura3 protein (above) expressed from the dicistronic reporter in $g c d 6-1$, GCD6 (left) and gcd7-201, GCD7 (right) yeast strains. The blot was stripped and reprobed with anti-PGK antibody as a loading control (bottom). (E) Northern analysis of total RNA using a probe to the $5^{\prime}$ end of the coding region of URA3 gene (top). An arrow indicates the full-length dicistronic message. Asterisk indicates a shorter transcript. The ethidium bromide staining of the $25 \mathrm{~S}$ rRNA serves as a loading control (bottom).

mutant strains, as indicated in Figure 1C. As a control, we used the LEU2 mutant IGR IRES (IGRmut) URA3 dicistronic reporter containing a two-nucleotide mutation in PK1 that yields a completely nonfunctional mutant CrPV IGR IRES (Wilson et al. 2000a; Thompson et al. 2001). Growth of the transformed strains was assayed on plates that contained $100 \mu \mathrm{M} \mathrm{CuSO}$ (to induce transcription of the dicistronic reporter), and IRES activity was assessed by comparing growth on plates with or without uracil. The level of IRES function is proportional to the level of growth on plates in the absence of uracil. As can be seen in Figure $1 C$, for both the $g c d 6-1$ and $g c d 7-201$ strains, the wild-type IGR IRES promoted growth on minus-uracil plates while the mutant IGR IRES did not. As we have shown previously, the CrPV IGR IRES does not function efficiently in wild-type yeast (Thompson et al. 2001). The growth observed in the absence of uracil correlated with the amount of Ura3p produced as demonstrated by Western analysis (Fig. 1D). Ura3p was produced in the $g c d 6-1$ and gcd7-201 strains expressing the wild-type IGR IRES (lanes $1,7)$, whereas no Ura3p was detected in GCD7 or GCD6 yeast, strains expressing the IGRmut (Fig. 1D, lanes
$2,5,8,11)$, or the vector pSall, which lacks the entire dicistronic cassette (Fig. 1D, lanes 3,6,9,12).

To determine whether full-length dicistronic reporter transcripts were produced in vivo we performed Northern analysis on RNA isolated from wild-type and mutant yeast strains with the IGR, IGRmut, or pSall plasmids (Fig. 1E). The dicistronic RNAs of the expected size were detected with a probe to the $5^{\prime}$ end of the URA 3 coding region in strains that contain the dicistronic reporter (IGR or IGRmut) but not in lanes with the empty vector (Fig. 1E, pSal1, lanes 3,6,9,12). Upon overexposure of the Northern, a faint smaller transcript was also observed (Fig. 1E, see asterisk). However, we will show in a following section that the shorter transcript contains the majority of the IGR IRES sequence and does not appear to use a capdependent mechanism to translate the URA3 coding region.

Taken together, these results demonstrate that the CrPV IGR IRES is significantly enhanced in vivo when eIF2B is mutated. This finding is consistent with the notion that a reduction in ternary complex (eIF2/GTP/Met-tRNA $\mathrm{A}_{\mathrm{i}}^{\mathrm{Met}}$ ) through eIF $2 \alpha$ phosphorylation, lowering of Met-tRNA $\mathrm{A}_{\mathrm{i}}^{\mathrm{Met}}$ (Thompson et al. 2001), or eIF2B inactivation (Fig. 1) increases CrPV IGR IRES activity in vivo. 


\section{A mutation in elF3b enhances IGR IRES activity}

During cap-dependent initiation, eIFs1, 3, and 5 and the ternary complex form a MFC that binds cooperatively to the $40 \mathrm{~S}$ ribosomal subunit. eIF3b (encoded by PRT1) is a component of eIF3, and the prt1-1 mutation impairs binding of all MFC components to the $40 \mathrm{~S}$ subunit in vitro (Phan et al. 2001). However, in vivo the rate-limiting defect for the prt1-1 mutation was found to be downstream from $48 \mathrm{~S}$ assembly prior to $60 \mathrm{~S}$ subunit joining, which includes scanning, AUG recognition, and GTP hydrolysis by the ternary complex (Nielsen et al. 2004). To determine whether the CrPV IGR IRES is affected by the prt1-1 mutation, we performed a growth assay with a strain harboring the prt1-1 mutation. Since prt1-1 is a temperature-sensitive mutant, we performed the growth assay at the intermediate temperature of $30^{\circ} \mathrm{C}$ to assess IGR IRES function in vivo. As can be seen in Figure 2A, the prt1-1 mutant strain exhibits a severe growth defect at $30^{\circ} \mathrm{C}$ on SD complete media, indicating that at this temperature Prtlp function is significantly impaired. Figure $2 \mathrm{~B}$ demonstrates that the IGR IRES promotes robust growth on plates lacking uracil in the prt1-1 but not in the wild-type yeast strain. In agreement with the growth assays, the Western analysis of Ura3p demonstrates that expression of Ura3p occurs only in the prt1-1 yeast strain when the wild-type IGR IRES is upstream of the URA3 ORF (Fig. 2C). Northern analysis confirmed that only full-length dicistronic transcripts are generated in these strains (Fig. 2D). Taken together, these results suggest that IGR IRESmediated translation is enhanced when eIF3b function is impaired.

\section{The IGR IRES functions efficiently when eIF4G levels are reduced in vivo}

Because some viral IRESs require the $\mathrm{C}$ terminus of eIF4G for efficient translation (Jackson 2005), we wanted to determine whether eIF4G is required for IGR IRES-mediated translation. In mammalian cells, eIF4G is thought to be important for recruiting the 43S PIC to the capped message through its interactions with eIF3 (Hershey and Merrick 2000). In yeast, however, an eIF3-eIF4G interaction has not been demonstrated, rather it has been suggested that an eIF5-eIF4G interaction promotes mRNA recruitment in yeast (Asano et al. 2001). For a subset of messages, eIF4G has been shown to have a rate-limiting step downstream from $48 \mathrm{~S}$ complex assembly, most likely during ribosome scanning (Jivotovskaya et al. 2006). eIF4G is essential in yeast and is encoded by two genes, TIF4631 and TIF4632; the encoded proteins eIF4G1 and eIF4G2, respectively, are functionally similar, and only one form is required for viability (Goyer et al. 1993). A strain lacking eIF4G1 exhibits a slight growth defect (Fig. 3A) and a cold-sensitive phenotype, while disruption of eIF4G2 failed to show any phenotype (Goyer et al. 1993). Therefore, to test for any significant effects from the depletion of eIF4G, we used a strain harboring a deletion in eIF4G1 to functionally reduce the amount of total eIF4G in the cell. Figure 3B demonstrates that the CrPV IGR IRES supports growth on minusuracil plates in the yeast strain lacking eIF4G1, indicating that it functions robustly to translate the URA3 ORF. Importantly, the mutant IRES that lacks IRES activity demonstrated no significant growth, suggesting that the growth observed is dependent on a functional IGR IRES. Western analysis of the Flag-tagged Ura3p from these strains demonstrates that only the eIF4G1-deleted strain with the wild-type IGR IRES results in Ura3p production (Fig. 3C, lane 1), consistent with the growth assays. In addition, Northern analysis reveals that full-length dicistronic mRNAs are produced in these strains (Fig. 3D). Taken together, these results suggest that the IGR IRES does not require eIF4G1 to initiate translation in vivo.

\section{A temperature sensitive mutation in elF4E enhances IGR IRES activity in vivo}

Typically IRESs do not require eIF4E; however, the hepatitis A virus IRES does (Ali et al. 2001). To determine whether eIF4E is required for CrPV IGR IRES function in vivo, we assayed IRES activity in a temperature-sensitive mutant, cdc33-ts (cdc33-4-2) (Altmann et al. 1989; Altmann and Trachsel 1997). This mutant, at the restrictive temperature of $37^{\circ} \mathrm{C}$, has $15 \%$ of wild-type translation (as measured by ${ }^{35} \mathrm{~S}$-methionine incorporation), demonstrates a reduced affinity for the mRNA cap analog $\mathrm{m}^{7} \mathrm{GDP}$, and fails to grow (Altmann et al. 1989). To test whether the IGR IRES requires eIF4E to initiate translation in vivo, we grew the yeast strains at an intermediate temperature of $27.5^{\circ} \mathrm{C}$, where we were able to observe a slight growth defect (Fig. 4A) but were still able to assay for IGR IRES activity using a growth assay that requires production of Ura3p by the IGR IRES to grow on a minus-uracil plate (Fig. 4B). We observed that the CrPV IGR IRES promoted translation of the URA3 ORF sufficient to confer growth on minusuracil plates. Consistent with the growth assays, the Flagtagged Ura3 protein was detected by Western analysis exclusively in the yeast strain harboring both the $c d c 33-t s$ mutant and the wild-type IGR IRES (Fig. 4C). Additionally, the Northern analysis revealed that predominantly fulllength dicistronic messages were produced (Fig. 4D, lanes $1,2,4,5)$. Taken together, these data indicate that IGR IRESmediated translation is enhanced in vivo when eIF4E is impaired.

\section{The CrPV IGR IRES does not require elF5B in vivo}

eIF5B facilitates 60S ribosomal subunit joining (Pestova et al. 2000). eIF5B is recruited to the 40 S subunit by eIF1A, and hydrolysis of eIF5B-GTP is important for release of 
A

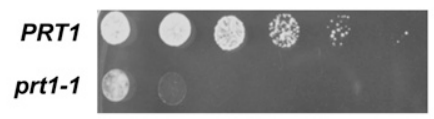

B
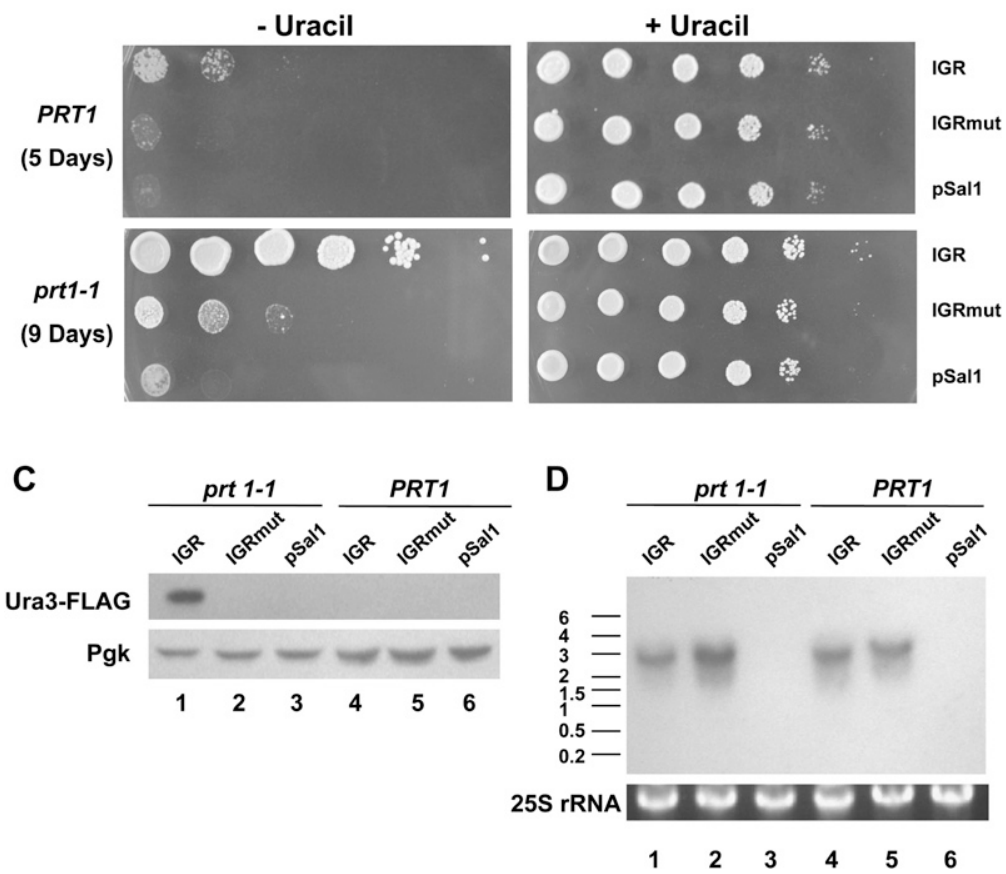

FIGURE 2. An eIF3 mutation enhances IGR IRES activity. (A) Growth assay on complete SD media shows relative growth phenotypes of wild-type (PRT1) vs. mutant (prt1-1) eIF3 strains. (B) Growth assay on SD media with $100 \mu \mathrm{M} \mathrm{CuSO}_{4}$ and supplemented without (left) or with (right) uracil. (C) Immunoblot using anti-Flag M2 monoclonal antibody to detect the Cterminal Flag tag of the Ura3 protein (top). The blot was stripped and reprobed with anti-PGK antibody as a loading control (bottom). (D) Northern analysis of total RNA using ${ }^{32} \mathrm{P}$ labeled DNA probe for the $5^{\prime}$ end of the URA3 coding region (top). The ethidium bromide staining of the $25 \mathrm{~S}$ rRNA serves as a loading control (bottom).

eIF1A and eIF5B from the $80 \mathrm{~S}$ monosome (Pestova et al. 2000; Fringer et al. 2007). Interestingly, eIF5B encoded by FUN12 is not an essential gene in yeast; however there is a severe slow growth phenotype (Fig. 5A, see fun $12 \Delta$ ). We used a fun $12 \Delta$ yeast strain to assess the role of eIF5B in CrPV IGR IRES-mediated translation. In the fun $12 \Delta$ strain transformed with the dicistronic reporter harboring the wild-type IGR IRES, we saw robust growth on minus-uracil plates (Fig. 5B), demonstrating that the CrPV IGR IRES does not require eIF5B for translation initiation. Importantly, there was no growth observed with the reporter containing the mutant IRES (IGRmut) or when the empty vector (pSal1) was present. As we have seen previously, no growth was observed for the wild-type yeast strain. Western analysis to detect the Flag-Ura3p supports the growth assay results, indicating that only the fun $12 \Delta$ strain with the wildtype IGR IRES results in production of Ura3p (Fig. 5C). Finally, only full-length dicistronic messages were detected (Fig. 5D). Taken together, these data demonstrate that translation of the IGR IRES is greatly enhanced in the eIF5BA strain and suggest that eIF5B is not required for IGR IRES activity.

\section{An excess of $40 S$ over 605 ribosomal subunits is not sufficient to promote IGR IRES-mediated translation in vivo}

The current results suggest that mutations that reduce the efficiency of capdependent initiation lead to enhanced activity of the IGR IRES, suggesting that cap-dependent and cap-independent mechanisms are in direct competition in vivo. Because the IGR IRES has been shown to bind to $40 \mathrm{~S}$ ribosomes in vitro (Wilson et al. 2000a; Jan and Sarnow 2002), we reasoned that it might be possible to enhance initiation by the CrPV IGR IRES in vivo if there were an excess of $40 S$ subunits in the cell. We tested IGR IRES activity in a yeast strain harboring a deletion of YNL069C (rpl16bs), one of the two genes coding for Rpl16p. A decrease in the levels of Rpl16p results in a reduction in the number of $60 \mathrm{~S}$ subunits in the cell, creating an excess of $40 \mathrm{~S}$ subunits relative to the $60 \mathrm{~S}$ subunits (Rotenberg et al. 1988). Growth on complete synthetic media at $30^{\circ} \mathrm{C}$ results in little or no growth defect (Fig. 6A). This growth defect is more pronounced at room temperature (data not shown). The growth assays revealed that in contrast to translation initiation factor mutants, no significant growth was observed for the rpl16bs strain transformed with the IGR IRES on plates without uracil (Fig. 6B). Western analysis of the Ura3p expression supports this result, as no Ura3p was observed for the rpll6bs strain (Fig. 6C). As a positive control, we included extracts from the fun $12 \Delta$ strain harboring the wild-type IGR IRES plasmid (Fig. 6C, lane 1 ). As we have shown previously in the fun $12 \Delta$ strain carrying the wild-type IGR IRES reporter, with the wildtype IGR IRES, a significant amount of Ura3p is generated (Figs. 6C, lane 1; 5C, lane 1). In contrast, no Ura3p is detected in the rpl16bs strain. We verified this finding with a sensitive dicistronic luciferase reporter in which we would be able to measure small changes in IGR IRES activity that may be undetectable by Western analysis or growth assays. The firefly luciferase values were not significantly different between the wild-type and rpl16bs strains (Fig. 6E). Since we did not observe any difference between the rpl16bs 
A

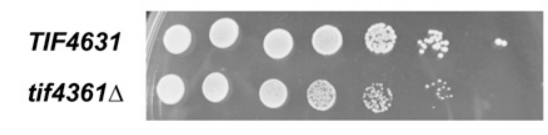

B

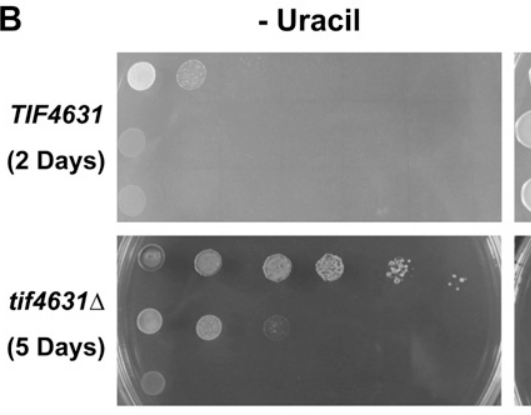

C

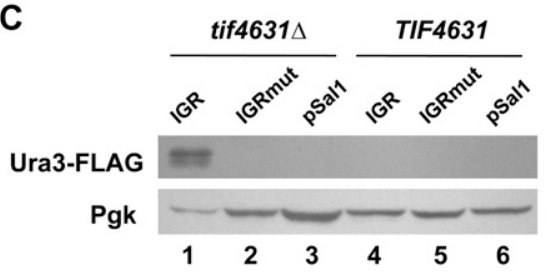

+ Uracil

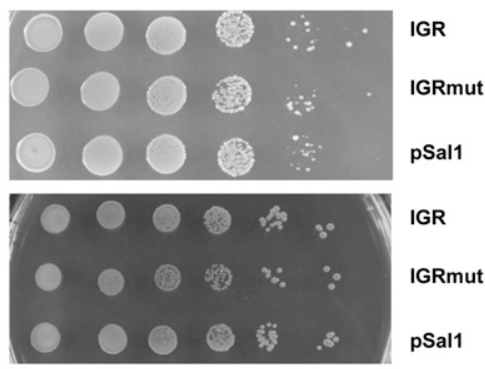

D

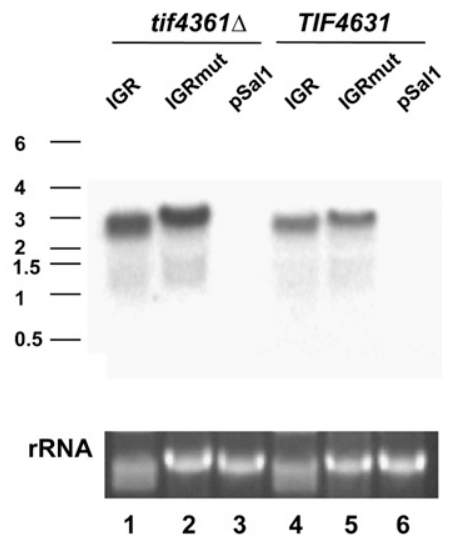

FIGURE 3. CrPV IGR IRES functions when eIF4G levels are reduced. (A) Growth assay on complete SD media shows relative growth phenotypes of wild-type (TIF4631) vs. mutant (tif4631) eIF4G1 strains. (B) Growth assay on SD media with $100 \mu \mathrm{M} \mathrm{CuSO}_{4}$ supplemented either without (left) or with (right) uracil. (C) Immunoblot using anti-Flag M2 monoclonal antibodies to detect the C-terminal Flag tagged Ura3 protein (top). The blot was stripped and reprobed with anti-PGK antibody as a loading control (bottom). (D) Northern analysis of total RNA using ${ }^{32} \mathrm{P}$ labeled DNA probe for the 5 ' end of the URA3 coding region (top). The ethidium bromide staining of the $25 \mathrm{~S}$ rRNA serves as a loading control (bottom).

strain and wild-type yeast, we confirmed by PCR analysis of the genomic DNA that the RPL16B gene is knocked out in the strain we are using (data not shown). In addition, we performed a polysome analysis of the rpll6bs strain and observed half-mer peaks (data not shown), which are characteristic of a defect in 605 joining and have been shown previously to be a characteristic of rpl16bs strains (Rotenberg et al. 1988). The polysome to monosome ratio was also decreased in the rpll6bs strain $(\mathrm{P} / \mathrm{M}=0.74)$ relative to wild type $(\mathrm{P} / \mathrm{M}=1.0)$, indicating that there is a defect in translation in the rpli6bs strain. Therefore, deletion of RPL16B does not enhance the IGR IRES activity in contrast to the affects we observed by decreasing or mutating translation initiation factors.

Northern analysis of the yeast strains confirmed that the full-length dicistronic mRNA was expressed in strains harboring the dicistronic LEU2 IGR URA3 reporter
(Fig. 6D). Upon overexposure, a smaller band was observed in these strains. However, we will demonstrate in the following section that the IGR IRES is present on this shorter transcript, and thus it is unlikely to result in capdependent expression of the Ura3 protein. Taken together, these results suggest that an excess of $40 \mathrm{~S}$ over $60 \mathrm{~S}$ subunits is not sufficient to enhance IRES activity in vivo.

\section{In vivo assay of IGR IRES activity is not affected by cap-dependent translation of shorter transcripts}

Upon overexposure of several of the Northerns, we observed the presence of a shorter transcript that could be a monocistronic message translated by cap-dependent initiation. The expected size of the dicistronic message is 2.8 $\mathrm{kbp}$, whereas a monocistronic message that could translate URA3 by a capdependent mechanism would be $\sim 1.2$ kbp. A monocistronic message that encoded the entire CrPV IGR IRES with the URA3 ORF would be $1.5 \mathrm{kbp}$, which is the size we consistently detected. The presence of the shorter transcript did not correlate with Ura3 protein expression as the shorter transcripts were consistently produced from both wildtype IGR and IGRmut dicistronic reporters. In contrast, only the wild-type IGR reporter exhibited Ura3 protein expression as assayed by growth assays and Western analysis. Taken together, these observations suggest that expression of the Ura3 protein is dependent on a functional IRES regardless of which transcript it is translated from.

To further substantiate this point, we used an oligo probe to determine whether the IGR IRES was present on the shorter transcript. Total RNA was isolated from $g c d 7$ 201 and GCD7 yeast strains harboring the dicistronic reporter with the IGR, IGRmut IRES, or the empty vector (pSal1). The Northern analysis was performed using an end-labeled IGR oligo probe to the core region of the IRES. The IGR oligo probe detected both the dicistronic transcript at just under $3 \mathrm{kbp}$ and the shorter transcript at 1.5 kbp (Fig. 7A), demonstrating that the IGR IRES is present on the shorter transcript. Similar results were found by Northern analysis of the $g c d 6-1$, tif $4631 \Delta$, and $r p l 16 b \Delta$, as well as their respective wild-type strains (data not shown). These results predict either a transcript that encodes LEU2, 
A

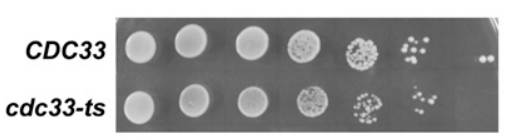

B
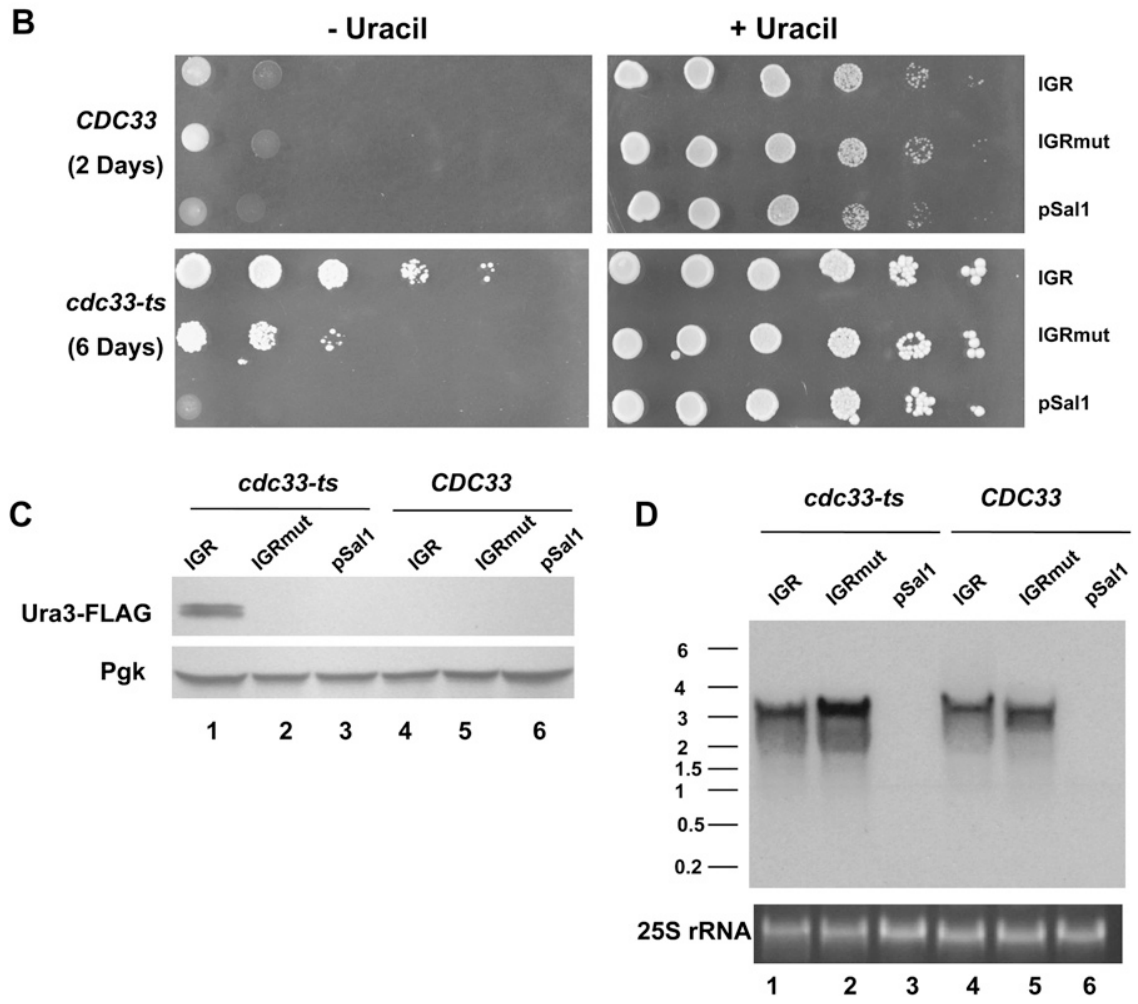

FIGURE 4. The IGR IRES can sustain growth in an eIF4E-ts mutant. (A) Growth assay on complete SD media shows relative growth phenotypes of wild-type (CDC33) and cdc33-4-2 mutant (cdc33-ts) eIF4E strains. (B) Growth assay on SD media with $100 \mu \mathrm{M} \mathrm{CuSO}_{4}$ without (left) or with (right) uracil. Strains were grown at $27.5^{\circ} \mathrm{C}$. (C) Immunoblot using anti-Flag M2 monoclonal antibody to detect the C-terminal Flag tagged Ura3 protein (top). The blot was stripped and reprobed with anti-PGK antibody as a loading control (bottom). (D) Northern analysis of total RNA using ${ }^{32} \mathrm{P}$ labeled DNA probe for the $5^{\prime}$ end of the URA3 coding region (top). Ethidium bromide staining of the $25 \mathrm{~S}$ rRNA serves as a loading control (bottom).

the IGR IRES, and the $5^{\prime}$ end of the URA 3 coding region or a transcript that encodes the IGR IRES and the URA3 coding region. The former would not generate a functional URA3p, while the latter is likely to be translated by a functional IGR IRES or not at all. Although we do not directly demonstrate that the small amount of monocistronic message is translated by the IGR IRES, it is unlikely to be translated by a cap-dependent mechanism. First, there are two start codons in the IGR IRES and multiple stop codons in all three reading frames. It is therefore unlikely that the shorter transcript would be translated by a capdependent mechanism. In addition, the Western analysis and the growth assays suggest that the Ura3 protein produced is IGR IRES-dependent, as Ura3 protein expression from the IGRmut IRES transcript was not observed and this mutation does not disrupt a start or stop codon or change the reading frame of the transcript relative to the wild-type IGR IRES. Furthermore, as previously mentioned, the presence of the shorter transcript does not correlate with Ura3 protein expression. We conclude that translation from the monocistronic RNA is either mediated by the CrPV IGR IRES or results in no production of Ura3p from this message.

It has also been proposed that several IRESs contain $3^{\prime}$ splice sites such that a small deletion undetectable by Northern blot could generate a fusion protein between the upstream $5^{\prime}$ cap-dependent ORF and the downstream ORF (Baranick et al. 2008). However, Western analysis clearly shows only a Flag-tagged URA3p at the expected molecular weight, and no evidence of a fusion protein was ever detected (data not shown). In addition, the wild-type IGR sequence is UUACCU GCU, which is changed by two nucleotides to generate UUAggUGCU for the nonfunctional IGRmut IRES. Given this small change in sequence, it is unlikely that a putative splice site was present in the wild-type sequence and disrupted in the mutant sequence.

To further evaluate the IGR IRES function in vivo, we generated a quantitative dicistronic luciferase reporter (Fig. 7B). To ensure that we were measuring only IGR IRES activity, we took advantage of the fact that firefly luciferase is sensitive to N-terminal truncations. Deletion of 10 amino acids from the $\mathrm{N}$ terminus reduces luciferase activity to less than $1 \%$ (Sung and Kang 1998). Therefore, deletion of the first AUG start codon results in a nonfunctional protein that would initiate at the next AUG codon 87 nucleotides downstream if a cap-dependent mechanism of translation is used. However, because the IGR IRES initiates at an alanine codon, if the IGR IRES is placed upstream of the $\triangle$ AUG firefly luciferase coding region, then a full-length, functional firefly luciferase protein is generated. Thus, the presence of shorter monocistronic transcripts would have no effect on the IGR IRES activity assayed using the $\triangle$ AUG firefly luciferase. We transformed wild-type, tif4631s, and fun $12 \Delta$ yeast strains with the dicistronic dual luciferase reporter and assayed for IRES activity. Consistent with the $\Delta$ AUG dicistronic luciferase reporter being unaffected by the presence of a monocistronic transcript, we obtain values similar to background levels (data not shown) with the IGRmut (Fig. 7C). The wild-type IGR IRES is active in both the tif4631s and fun12s yeast strains exhibiting 
A
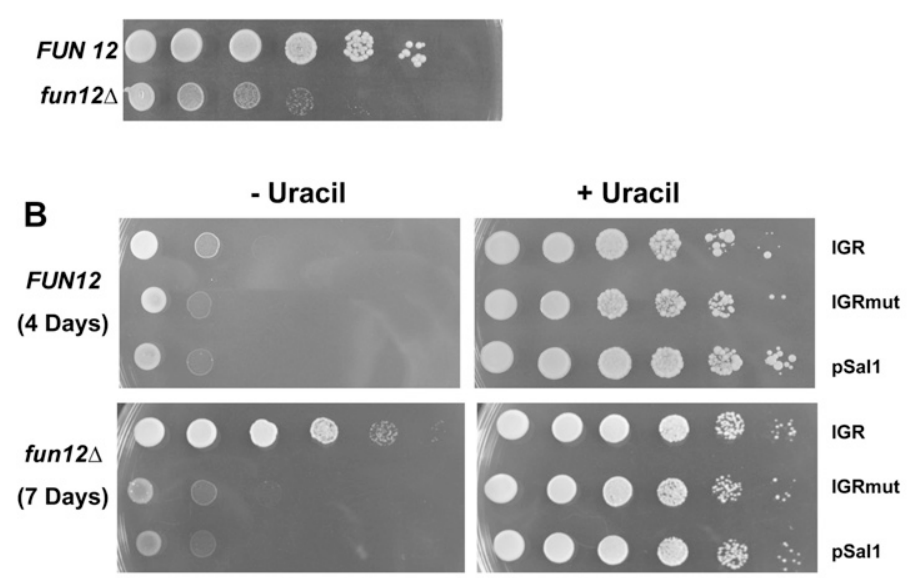

C

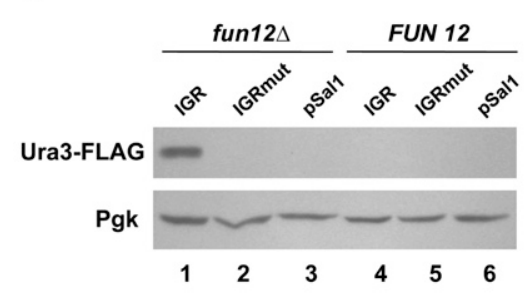

25S rRNA
D

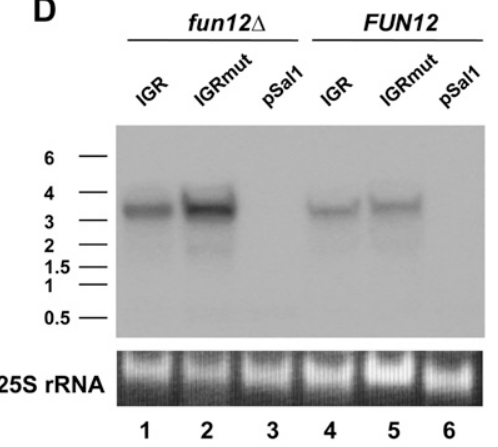

FIGURE 5. CrPV IGR IRES does not require eIF5B in vivo. (A) Growth assay on complete SD media shows relative growth phenotypes of wild type (FUN12) and a mutant strain lacking eIF5B (fun12A). (B) Growth assay on SD media with $100 \mu \mathrm{M} \mathrm{CuSO}{ }_{4}$ supplemented either without (left) or with (right) uracil. (C) Immunoblot using anti-Flag M2 monoclonal antibodies to detect the C-terminal Flag tagged Ura3 protein (top). The blot was stripped and reprobed with anti-PGK antibody as a loading control (bottom). (D) Northern analysis of total RNA using ${ }^{32} \mathrm{P}$ labeled DNA probe for the $5^{\prime}$ end of the URA3 coding region (top). The ethidium bromide staining of the $25 \mathrm{~S}$ rRNA serves as a loading control (bottom).

ninefold and 24-fold more firefly luciferase activity, respectively, compared with the isogenic wild-type yeast strain (Fig. 7C). Therefore, these results agree with those obtained using the LEU2 IGR URA3 reporter. Interestingly, the wildtype IGR IRES is active in wild-type cells, yielding $\sim 10$-fold more activity compared with background levels (see Fig. $6 \mathrm{E})$. However, the IGR IRES activity is not sufficient to support growth in wild-type yeast when it drives expression of a selectable marker, nor is this level of expression detectable by Western analysis. In addition, we never detected any Ura3 protein in samples from yeast harboring the IGRmut IRES, consistent with the fact that firefly luciferase assays of strains harboring IGRmut IRES dicistronic reporter always resulted in background levels of firefly luciferase (Fig. 7C; Table 1, below). Since capdependent translation is affected to varying degrees in the three strains, wild type, tif $4631 \Delta$, and fun12s, we are unable to normalize the firefly values to the cap-dependent translation of Renilla luciferase. The same amount of yeast was harvested for each assay. We observed a $30 \%$ decrease in Renilla luciferase activity for the fun $12 \Delta$ strain relative to wild-type yeast (Table 1), which is consistent with the amount of decrease observed in total protein synthesis when eIF5B was depleted in yeast in vivo (Sangthong et al. 2007). Thus, a small decrease in cap-dependent translation can translate into a large enhancement of IGR IRES-mediated translation (Fig. 7C). We conclude that the IGR IRES functions robustly in vivo in the absence of initiation factors and in wild-type cells at a reduced level.

\section{DISCUSSION}

We demonstrate that the IGR IRES functions most efficiently when initiation factors are limiting, and suggest that eIF4E, eIF2B, eIF5B, eIF4G1, and the eIF3b subunit of eIF3 are not required for CrPV IGR IRES-mediated translation initiation in vivo. The IGR IRES is still capable of initiating translation in wild-type cells at a significantly reduced level. Our in vivo data validate the physiological relevance of the model that the IGR IRES is able to directly recruit ribosomes, which was established based on in vitro biochemical assays.

In vitro binding assays indicate that ternary complexes plus eIF3 severely inhibit IGR IRES binding to $40 \mathrm{~S}$ subunits, whereas eIF1, eIF1A, and eIF3 alone or in combination had very little effect. This suggests that the IGR IRES is unable to bind to the $40 \mathrm{~S}$ subunit when a ternary complex is already bound to the $40 \mathrm{~S}$ subunit (Pestova et al. 2004). This is consistent with our observations in vivo that mutations in $g c d 6-1, g c d 7-201$ (Fig. 1), deletion of initiator Met-tRNA $\mathrm{i}_{\mathrm{i}}^{\mathrm{Met}}$ genes, or expression of a constitutively active GCN2 (Thompson et al. 2001) resulted in an enhancement of IGR IRES activity; all of these mutants result in a decrease in ternary complex in the cell. These data support a model in which the CrPV IGR IRES is unable to function robustly in wild-type cells because it is unable to compete effectively with the ternary complex for binding to the Psite of the $40 \mathrm{~S}$ ribosomal subunit. Since ternary complex binding to the $40 \mathrm{~S}$ subunit is enhanced by eIF1A, eIF1, eIF3, and eIF5 (Majumdar et al. 2003; Kolupaeva et al. 2005; Hinnebusch et al. 2007), we would expect that any mutation within this complex that results in a reduction of ternary complex binding would enhance the IGR IRES activity. 
A

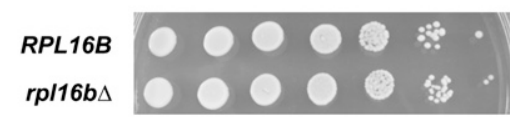

B
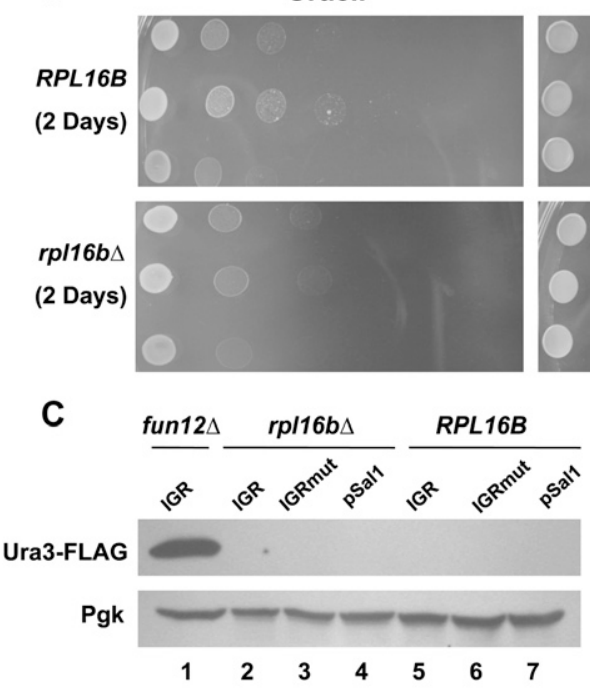

E

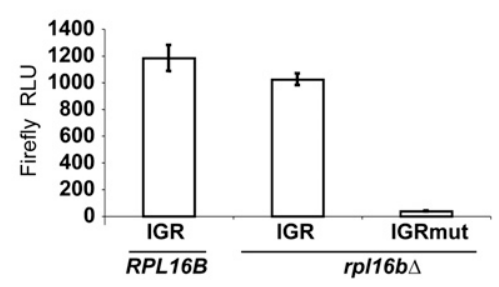

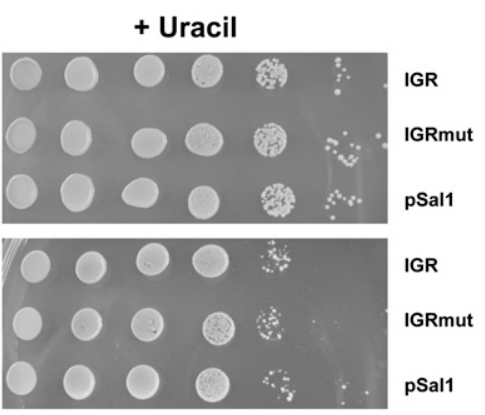

D

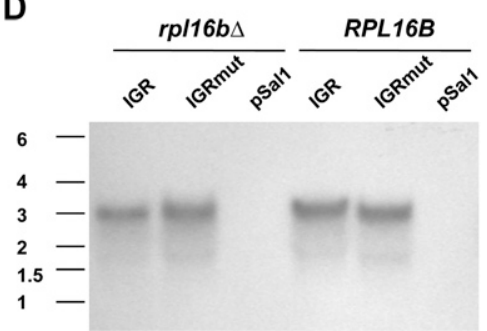

$25 \mathrm{~S}$ rRNA

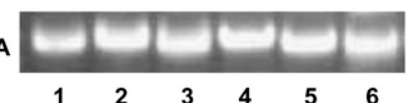

FIGURE 6. An excess of 40 S over 60 S subunits is not sufficient to enhance IGR IRES function. (A) Growth assay on complete SD media showing relative growth phenotypes of wild-type (RPL16B) and ribosomal mutant (rpl16bs) strains. (B) Growth assay on SD media with 100 $\mu \mathrm{M} \mathrm{CuSO}_{4}$ and either without (left) or with (right) uracil. (C) Immunoblot using anti-Flag M2 monoclonal antibodies to detect the C-terminal Flag tagged Ura3 protein (top). The fun $12 \Delta$ yeast strain with the IGR dicistronic reporter is shown as a positive control. The blot was stripped and reprobed with anti-PGK antibody as a loading control (bottom). (D) Northern analysis of total RNA using ${ }^{32} \mathrm{P}$ labeled DNA probe for the $5^{\prime}$ end of the URA3 coding region (top). The ethidium bromide staining of the 25S rRNA serves as a loading control (bottom). (E) Luciferase assays of $R P L 16 B$ or $r p l 16 b \Delta$ harboring a dicistronic luciferase reporter with either a wild-type or mutant IGR IRES.

While the addition of eIF1, eIF1A, and eIF3 has little or no effect on IGR IRES binding to $40 \mathrm{~S}$ subunits, the formation of $80 \mathrm{~S}$ complexes is inhibited in vitro. This inhibition can be relieved through the addition of eIF5B (Pestova et al. 2004). These results suggest that eIF5B may be functioning to release eIF1A, eIF1, and eIF3 from the $48 \mathrm{~S}$ complex (43S with IRES) in order for the $60 \mathrm{~S}$ subunit to join in vitro. In fact, subsequent in vitro studies have shown that during cap-dependent translation eIF1A recruits eIF5B to the $40 \mathrm{~S}$ subunit to facilitate subunit binding, then GTP hydrolysis enables the release of eIF1A and eIF5B before the elongation phase begins (Pestova et al. 2004; Fringer et al. 2007). In contrast, our data clearly demonstrate that a deletion in eIF5B enhances IGR IRES activity in vivo (Fig. 5B,C). These contradictory results can be reconciled if eIF1, eIF1A, and eIF3 do not form a complex with the IGR IRES in vivo; however when this complex is formed in vitro, eIF5B is required to release these factors in order for subunit joining to occur.

We demonstrate that the CrPV IGR IRES does not require eIF5B, suggesting that subunit joining can occur in the absence of eIF5B. It is notable that the CrPV IGR IRES activity was enhanced to the greatest extent by the eIF5B deletion (Figs. 5B, 7C). There could be several possible explanations for this. First, the fun $12 \Delta$ yeast strains exhibit the most severe growth defects, suggesting that cap-dependent translation initiation is impaired more severely in these cells. This supports the notion that the more cap-dependent translation is decreased, the better the IGR IRES is able to compete for ribosomes. Second, the IGR IRES may have a mechanism to efficiently promote $60 \mathrm{~S}$ joining or may bypass this step in vivo by binding directly to $80 \mathrm{~S}$ subunits, as has been suggested in vitro (Pestova et al. 2004). The cryo-EM studies indicate that CrPV IGR IRES binding induces a conformational change to the $40 \mathrm{~S}$ ribosomal subunit (Spahn et al. 2004; Schuler et al. 2006), which may alter the conformation such that the $60 \mathrm{~S}$ subunit can join efficiently in the absence of eIF5B. Finally, eIF5B may compete with the IGR IRES for interactions with the ribosome during subunit joining.

The 80 S complexes bound to the IGR IRES can become translationally competent with the addition of elongation factors eEF2, eEF1A, and aminoacylated tRNAs to generate a small peptide in vitro, although not under physiological conditions (Jan et al. 2003; Pestova and Hellen 2003). These experiments raised the question of whether the CrPV IGR IRES utilized translation initiation factors in vivo. Our results suggest that, the CrPV IGR IRES initiates translation in vivo independent of the initiation factors eIF4E, eIF4G, eIF2B, eIF5B, and eIF3. This is in agreement with the in vitro assays that suggest the IGR IRES can initiate translation in the absence of any initiation factors (Jan et al. 2003; Pestova and Hellen 2003).

We used the rpl164 yeast strain, which has a defect in $60 S$ ribosomal subunit assembly to demonstrate that an excess of $40 \mathrm{~S}$ over $60 \mathrm{~S}$ subunits is unable to enhance the 
TABLE 1. Dicistronic luciferase assay data

\begin{tabular}{lllr}
\hline Yeast & IRES & \multicolumn{1}{c}{ Renilla RLU } & Firefly RLU \\
\hline Wild type & IGR & $383,032 \pm 39,450$ & $1,858 \pm 375$ \\
& IGRmut & $436,579 \pm 101,956$ & $136 \pm 54$ \\
tif46314 & IGR & $519,390 \pm 8,598$ & $17,167 \pm 377$ \\
& IGRmut & $486,278 \pm 19,913$ & $70 \pm 2$ \\
fun124 & IGR & $286,032 \pm 24,701$ & $45,379 \pm 3,193$ \\
& IGRmut & $281,797 \pm 14,027$ & $166 \pm 38$ \\
\hline
\end{tabular}

IGR IRES activity in vivo. Presumably these cells still contain the full compliment of initiation factors. Therefore, the $40 \mathrm{~S}$ subunits are not in excess over the initiation factors such that the $40 \mathrm{~S}$ subunits are fully occupied by ternary complexes, which may compete for IGR IRES binding. Since the biochemical and structural data suggest that the PK I structure of the IGR IRES is located in the P-site of the ribosome, the IGR IRES may compete directly with ternary complex binding to the $40 \mathrm{~S}$ subunit. In fact, several studies have suggested that the IGR IRES itself mimics the initiator Met-tRNA $_{i}^{\text {Met }}$ and elongator tRNA in the ribosomal P-site (Jan et al. 2003; Pestova and Hellen 2003; Costantino et al. 2008). Alternatively, the absence of an effect on IGR IRES activity in the rpllobs strain may be due to the fact that this is the weakest translation mutation we tested and that deletion of another translation factor that yielded a more significant growth defect would result in enhancement of the IGR IRES. However, at the temperatures that we grew the eIF4E mutants (Fig. 4A), we observed very little growth difference, if any, but a significant enhancement of IGR IRES activity. If the affect of the rpl16bs were proportional to the affect on translation, then we would have expected to detect some enhancement of the IGR IRES in the rpll6bs strain with the sensitive luciferase assay; however, we did not observe this. Interestingly, decreases in specific ribosomal proteins have been shown to both decrease (Cherry et al. 2005) and increase (Barna et al. 2008) IRES activity.

In many cases, as we have shown here, cap-dependent translation must be reduced in order to enhance IRES activity. Our findings are consistent with a model in which IRESs are unable to recruit ribosomes efficiently unless capdependent translation is attenuated. This model predicts that there is a competition between cap-dependent and cap-independent translation. Similarly, during cellular stresses such as hypoxia, starvation, viral infection, and apoptosis, translation initiation factors are targeted for modification or cleavage in order to inhibit cap-dependent translation. For example, kinases such as PKR, PERK, and GCN2 phosphorylate eIF2 $\alpha$ during cell stress. This competition would allow the cell to globally shut down protein synthesis while enhancing the translation of IREScontaining mRNAs that encode proteins necessary for the cell to cope with the stress. Indeed, it is under conditions of cellular stress or conditions when cap-dependent translation is reduced, such as mitosis, that most cellular IRESs have been shown to function. In our system, we decreased cap-dependent translation by deleting or impairing translation initiation factors, resulting in an enhancement of IGR IRES activity. From our experiments, it is clear that impairing either early or late steps in translation initiation results in an enhancement of IGR IRES function. This observation is consistent with the in vitro data that suggest that the IGR IRES binds directly to $40 \mathrm{~S}$ and 60 S ribosomes to form $80 \mathrm{~S}$ complexes that are competent for elongation (Jan et al. 2003).

We have presented data that demonstrate that the IGR IRES activity is enhanced in vivo in multiple mutants that are impaired at various steps in translation initiation resulting in distinct $40 \mathrm{~S}$ complexes. For example, a reduction in ternary complexes would generate free $40 \mathrm{~S}$ subunits, while deletion of eIF5B results in mRNAs with a $40 \mathrm{~S}$ subunit bound waiting for a 605 subunit to join. Additionally, the prt1-1 and $c d c 33$-ts mutants do not alter $48 \mathrm{~S}$ complex formation. Therefore, if $40 \mathrm{~S}$ binding was the ratelimiting step for IGR IRES function in vivo, then we would

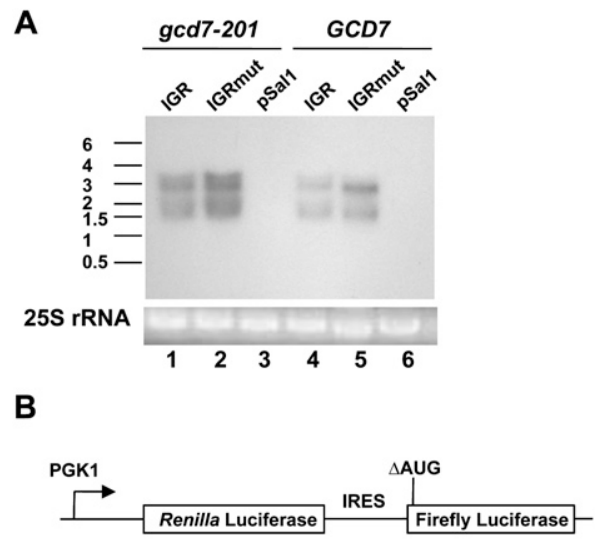

C

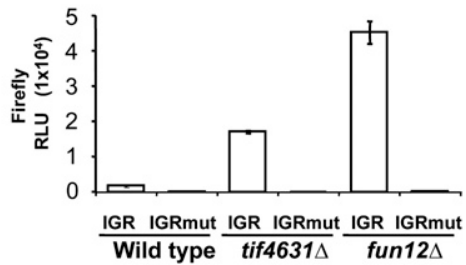

FIGURE 7. In vivo IGR IRES activity is independent of any contributions by a cap-dependent mechanism of translation. (A) Northern analyses of the dicistronic reporter transcripts from $g c d 7$ 201 and GCD7 yeast strains using a ${ }^{32} \mathrm{P}$-labeled oligo probe against the core region (SL2.1) of the CrPV IGR IRES (top). The ethidium bromide staining of the $25 \mathrm{~S}$ rRNA serves as a loading control (bottom). (B) A diagram of the $\triangle \mathrm{AUG}$ dicistronic luciferase reporter.

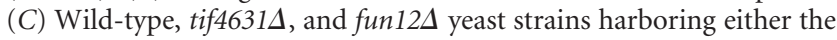
IGR or IGRmut IRES $\triangle$ AUG dicistronic luciferase reporter. Graph illustrating the firefly luciferase activity standard error is indicated for $\mathrm{n}=3$. 
Deniz et al.

TABLE 2. Primers used in this study

\begin{tabular}{ll}
\hline Name & \\
\hline IGR_FWD & \multicolumn{1}{c}{ Sequence $\left(5^{\prime}\right.$ to $\left.3^{\prime}\right)$} \\
IGR_REV & GAATTCGCTAGCCTCGAGGCAAAAATGTGATCTTGCTTGTA \\
RLucFWD & GCATGCAGATCTCCATGGAATCTTGAAATGTAGCAGGTAAAT \\
RLucREV & GCTAGCATGACTTCGAAAGTT \\
FLucFWD & GAGCTCTTATTGTTCATTTTTGAGAACTCGCTCAA \\
FLuCREV & CCATGGAAATGGAAGACGCCAAAAACATA \\
PGK1_FWD & AGATCTTTACACGGCGATCTTTCC \\
PGK1_REV & AATGTCAGGATCCAAACCCAGACACGCTCGACTTCCTG \\
ReConstructed_IGR_FWD & CATGGTGGCTAGCTTTGTTTTATATTTGTTGTAAAAAGTAG \\
Reconstructed_IGR_REV & GCTAAATACTCGAGAGCAAAAATGTGATCTTGC \\
$\Delta$ AUG_SENSE & CGAAACCATGGTATCTTGAAATGTAGCAGGTAAATTTC \\
$\Delta$ AUG_ANTISENSE & TTACCTGCTACATTTCAAGATTCCATGACCATGGAAGACGCCAAAAACATAAA \\
$\Delta$ PKI_SENSE & TTTCTTTATGTTTTTGGCGTCTTCCATGGTCATGGAATCTTGAAATGTAGCAG \\
$\Delta$ PKI_ANTISENSE & CAGATTAGGTAGTCGAAAAACCTAAGAAATTAGGTGCTACATTTCAAGATT \\
IGR oligo probe & AATCTTGAAATGTAGCACCTAAATTTCTTAGGTTTTTCGACTACCTAATCTG \\
\hline
\end{tabular}

not necessarily expect to see enhancement of the IGR IRES in the eIF5BA strain, but we clearly do. Therefore, in order to explain enhancement of IGR IRES activity in all of these mutants, we looked for shared characteristics between all of these mutants. All the strains we tested, including the rpl16bs strain, have a reduced polysome to monosome ratio, indicating an impairment of translation initiation.

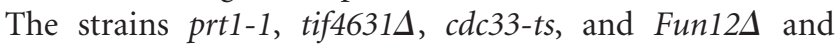
strains that have a reduced amount of ternary complexes accumulate $80 \mathrm{~S}$ complexes in contrast to the rpl16bs strain, which does not accumulate $80 \mathrm{~S}$ complexes and does not enhance IGR IRES activity (Lee et al. 2002; Nielsen et al. 2004; Jivotovskaya et al. 2006). In vitro studies demonstrated that the IGR IRES is capable of directly binding to $80 S$ complexes in vitro (Pestova et al. 2004). Therefore, the
IGR IRES may be able to directly associate with the accumulated $80 \mathrm{~S}$ complexes to initiate translation in vivo.

Although the CrPV IGR IRES is the simplest IRES with respect to factor requirement, it serves as a good model IRES for understanding this complex mechanism of initiation. It is likely that the diverse array of viral and cellular IRESs share some basic conserved mechanisms of initiation as they interact with the highly conserved ribosomes within the cell. Our results demonstrate that the IGR IRES is similar to other viral and cellular IRESs, in that cap-dependent translation must be impaired for the IRES to be able to recruit ribosomes efficiently. It has been suggested the IRESs evolved first, in that they are able to bind to ribosomes and establish a reading frame independent of other factors (Hernandez 2008). With time, other factors

TABLE 3. Yeast strains used in this study

\begin{tabular}{|c|c|c|}
\hline Strain & Genotype & Source \\
\hline F694 & MATa cdc33::LEU2 ade2 his3 leu2 trp1 ura3 & Altmann et al. (1989) \\
\hline F695 & MATa cdc33::LEU2 ade2 his3 leu2 trp1 ura3 & Altmann et al. (1989) \\
\hline F729 & MATa his3- $\Delta 1$ leu2- $\Delta O$ met15- $\Delta O$ ura3- $\Delta O$ & Winzeler et al. (1999) \\
\hline F885 & $\begin{array}{l}\text { MATa his3- } \Delta 1 \text { leu2- } \Delta 0 \text { met15- } \Delta 0 \text { ura3- } \Delta 0 \\
\quad \Delta \text { fun12::KanMX4 }\end{array}$ & Winzeler et al. (1999) \\
\hline F1048 & $\begin{array}{l}\text { MATa rp/16b::KanMX4 his3- } \Delta 1 \text { leu2- } \Delta 0 \text { met15- } \Delta 0 \\
\quad \text { ura3- } \Delta 0\end{array}$ & Winzeler et al. (1999) \\
\hline F1077 & $\begin{array}{l}\text { MATa his3- } \Delta 1 \text { leu2- } \Delta 0 \text { met15- } \Delta 0 \text { ura3- } \Delta 0 \\
\quad \text { tif4631::KanMX4 }\end{array}$ & Winzeler et al. (1999) \\
\hline H1515 & MATa ura3-52 leu2-112 trp1- 463 & Marton et al. (1993) \\
\hline$J 111$ & 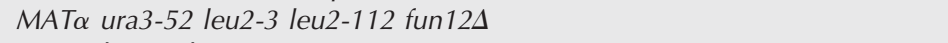 & Choi et al. (2000) \\
\hline H1730 & MAT $\alpha$ leu2-3 leu2-112 ura3-52 & Bushman et al. (1993) \\
\hline H1729 & MATa gcd6-1 leu2-3 leu2-112 ura3-52 & Alan Hinnebusch, NIH (unpubl.) \\
\hline $\mathrm{H} 1726$ & 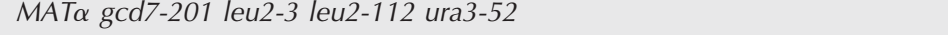 & Alan Hinnebusch, NIH (unpubl.) \\
\hline H1727 & МАТ $\alpha$ leu2-3 leu2-112 ura3-52 & Bushman et al. (1993) \\
\hline H1676 & MATa prt1-1 leu2-3 leu2-112 ura3-52 & Phan et al. (1998) \\
\hline $\mathrm{H} 2879$ & MATa leu2-3 leu2-12 ura3-52 & Nielsen et al. (2004) \\
\hline
\end{tabular}


including IRES transacting factors (ITAFs) and initiation factors became integrated into translation. This change presumably enhanced the efficiency of translation initiation, which is the rate-limiting step in protein synthesis, and allowed for an increased opportunity for regulation.

In conclusion, our data support a model in which the CrPV IGR IRES uses an initiation factor-independent mechanism of initiation in vivo. We have observed that the IGR IRES is only minimally active in wild-type yeast but initiates translation robustly when cap-dependent translation is impaired. Furthermore, we have identified several conditions under which the CrPV IGR IRES activity is enhanced in vivo. Our results suggest that the IGR IRES is unable to compete effectively with the initiation factors that work in concert to recruit ribosomes for cap-dependent translation. We suggest a possible mechanism in which the IGR IRES may directly associate with $80 \mathrm{~S}$ complexes in vivo.

\section{MATERIALS AND METHODS}

\section{DNA plasmids}

Wild-type and mutant dicistronic reporter plasmids IGR ( $\mathrm{pLEU2}$ IGR URA3) and IGRmut (pLEU2 IGRmut14 URA3) were previously published (Thompson et al. 2001). The dicistronic reporters were cloned into the pSal5 vector containing the HIS3 gene (a generous gift from J.O. Mascorro-Gallardo, Universidad Nacional Autónoma de México) to generate p5LEU2 IGR URA3 and p5LEU2 IGRmut14 URA3. Briefly, they were constructed by inserting the PvuI fragment from LEU2 IGR URA3 or LEU2 IGRmut14 URA3 containing the dicistronic reporter cassette into the pSal5 vector. The dicistronic luciferase plasmid was constructed using the pSall vector backbone (Mascorro-Gallardo et al. 1996). The CrPV IGR IRES was PCR amplified with the IGR_FWD and IGR_REV primers (Table 2) and cloned into the pSall vector using the EcoRI-BglII sites, creating pSalI-IGR. The Renilla and firefly luciferases were each amplified individually by PCR from the dicistronic luciferase plasmid (Wilson et al. 2000b) using the RlucFWD and RLucREV and the FlucFWD and FLucREV primers, respectively. The PCR products were cloned into the pSall-IGR backbone using the NheI-XhoI and NcoI-BglII sites, respectively, generating the pSall dual-luc IGR plasmid. The PGK1 promoter was PCR amplified with PGK1_FWD and PGK1_REV primers and replaced the BamHI and NheI fragment containing the CUP1 promoter of pSall dual-luc IGR, creating the PGK1 dual-luc IGR plasmid. The region upstream and downstream from the CrPV IGR IRES was reconstructed by PCR amplifying the IGR IRES using the reconstructed_IGR_FWD and reconstructed_IGR_REV primers, and replacing the XhoI-NcoI fragment from the PGK1 dual-luc IGR plasmid with the new IGR IRES that added a single adenosine at the $5^{\prime}$ end and the first five codons of CrPV ORF2 at the $3^{\prime}$ end, followed by one codon encoding threonine before the firefly ORF. The deletion of the firefly luciferase start codon was generated using the Quickchange mutagenesis kit (Stratagene) with the $\triangle \mathrm{AUG}$ _SENSE and $\triangle \mathrm{AUG}$ ANTISENSE primers. The CC-to-GG mutation (IGRmut) was also generated using the Quickchange mutagenesis kit (Stratagene) with the $\triangle \mathrm{PK} 1 \_$SENSE and $\triangle \mathrm{PK} 1 \_$ANTISENSE primers. The promoter and the entire dicistronic message sequences were confirmed by sequencing.

\section{Yeast strains and genetic methods}

Standard methods were used for culturing and transforming yeast strains (Ausubel et al. 1989). Untransformed strains were grown on YPD; transformed strains were grown on synthetic media. Yeast strains used in this study are listed in Table 3. Strains H1729 and $\mathrm{H} 1726$ are genetically identical to the published strains H1728 and H1725, respectively (Bushman et al. 1993).

\section{Growth assays}

Yeast strains harboring dicistronic reporters or the empty vector (pSal1 or pSal5) were grown at $30^{\circ} \mathrm{C}$ unless otherwise noted $\left(27.5^{\circ} \mathrm{C}\right.$ for $\mathrm{F} 694$ and $\left.\mathrm{F} 695\right)$ in $\mathrm{SD}$ media supplemented with the required amino acids, uracil, and $100 \mu \mathrm{M} \mathrm{CuSO}_{4}$ to early log phase. Equal $\mathrm{OD}_{600}$ units were centrifuged for $1 \mathrm{~min}$ at $16,000 \mathrm{~g}$, washed with SD media lacking uracil, resuspended in SD media lacking uracil, and incubated for $30 \mathrm{~min}$ at $30^{\circ} \mathrm{C}$ with shaking to deplete endogenous uracil pools. Tenfold serial dilutions were spotted onto SD plates with the required amino acids, with or without $100 \mu \mathrm{M}$ $\mathrm{CuSO}_{4}$ (as indicated), and incubated at $30^{\circ} \mathrm{C}$ (unless otherwise indicated). To demonstrate the relative growth defects imparted by the initiation factor mutations, serial dilutions of yeast strains transformed with pSal1 or pSal5 (vector) were spotted onto SD complete media (SD supplemented with leucine, lysine, uracil, adenine, tryptophan, methionine, and histidine amino acids).

\section{Immunoblot analysis}

Yeast strains were grown in SD media supplemented with uracil and $100 \mu \mathrm{M} \mathrm{CuSO}_{4}$ to an $\mathrm{OD}_{600}$ of 1.0. Cells were harvested from $100 \mathrm{~mL}$ cultures by centrifugation at $4^{\circ} \mathrm{C}$ at $1500 \mathrm{~g}$ for $5 \mathrm{~min}$ and washed with $\mathrm{ddH}_{2} \mathrm{O}$. Cells were disrupted by vortexing in three volumes glass bead disruption buffer $(20 \mathrm{mM}$ Tris at $\mathrm{pH} 7.9,10$ $\mathrm{mM} \mathrm{MgCl}_{2}, 1 \mathrm{mM}$ EDTA, 5\% glycerol, $1 \mathrm{mM}$ DTT, $0.3 \mathrm{M}$ ammonium sulfate, and $1 \mathrm{mM}$ PMSF) and four volumes of acidwashed, chilled glass beads (Sigma). Lysates were cleared by centrifuging at $12,000 \mathrm{~g}$ for $60 \mathrm{~min}$. SDS-PAGE analysis of $20 \mu \mathrm{g}$ of protein was transferred to Immobilon-P (Millipore). Anti-Flag M2 antibody (Sigma) was used to visualize the Flag-epitope-tagged Ura3 protein. The mouse anti-PGK monoclonal antibody (clone 22C5; Invitrogen) was used to detect 3-phosphoglycerate kinase. The goat anti-mouse (Santa Cruz Biotechnology) was used as the secondary antibody. Immunoblots were developed using an ECL kit (Pierce), as directed by the manufacturer.

\section{Northern analysis}

Yeast strains were grown at $30^{\circ} \mathrm{C}$ (or $27.5^{\circ} \mathrm{C}$ for $\mathrm{F} 694$ and $\mathrm{F} 695$ ) in $\mathrm{SD}$ media supplemented with uracil and $100 \mu \mathrm{M} \mathrm{CuSO}_{4}$ to an $\mathrm{OD}_{600}$ of 1.0. Cells were harvested from a $10-\mathrm{mL}$ culture by centrifugation for $10 \mathrm{~min}$ at $4^{\circ} \mathrm{C}$ at $1500 \mathrm{~g}$ and washed once with $\mathrm{ddH}_{2} \mathrm{O}$. Cell pellets were resuspended in $300 \mu \mathrm{L}$ RNA buffer $(0.5$ $\mathrm{M} \mathrm{NaCl} / 200 \mathrm{mM}$ Tris- $\mathrm{Cl}$ at $\mathrm{pH} 7.5 / 10 \mathrm{mM}$ EDTA) and then 300 $\mu \mathrm{L}$ acid phenol (5:1 phenol:chloroform at $\mathrm{pH} 4.5$, Ambion), and $200 \mu \mathrm{L}$ acid-washed glass beads were added. The cells were 
disrupted by vortexing for $2 \mathrm{~min}$. The aqueous phase was separated by centrifugation for $2 \mathrm{~min}$ at $11,000 \mathrm{~g}$. The aqueous phase was removed and re-extracted with an equal volume of acid phenol and precipitated with 3 volumes of $100 \%$ ethanol. Ten micrograms of total RNA was separated on a $0.8 \%$ agarose formaldehyde MOPS (0.4 M MOPS, $0.1 \mathrm{M} \mathrm{NaOAc}, 10 \mathrm{mM}$ EDTA) gel, and transferred to a Zeta probe membrane (Bio-Rad). The membrane was probed with ${ }^{32} \mathrm{P}$-labeled StuI/XbaI fragment of URA3 or an end-labeled IGR oligo probe (Table 2) and analyzed by autoradiography.

\section{Dual luciferase assays}

Yeast transformed with dicistronic luciferase plasmids were prepared for luciferase assays by growing overnight in selective media; $1 \mathrm{OD}_{600}$ of yeast was harvested in mid-log phase and resuspended in $100 \mu \mathrm{L}$ for $2 \mathrm{~min}$ in Passive Lysis Buffer (Promega). Luciferase assays were performed using the Dual-Luciferase Reporter Assay System (Promega) according to manufacturer's protocols. Activity was measured using a Lumat LB9507 luminometer (Berthold). Data represent the mean \pm SE $(n=3)$.

\section{ACKNOWLEDGMENTS}

We thank David Bedwell and R. Curtis Hendrickson for critical reading of the manuscript, and Cathy Galloway for her expertise as an editor. We thank Tom Dever and Alan Hinnebusch for generously providing yeast strains used in this study, and thank Tom Dever for many helpful discussions. We also thank J.O. Mascorro-Gallardo for providing us with the pSal5 plasmid. This work was supported by grants AI57560 (to S.R.T), 5T32AI007150 (to E.M.L), and 5T32HL007553 (to D.M.L) from the National Institutes of Health. The content is solely the responsibility of the authors and does not necessarily represent the official views of the National Heart, Lung, and Blood Institute, National Institute of Allergy and Infectious Diseases, or the National Institutes of Health.

Received August 13, 2008; accepted January 27, 2009.

\section{REFERENCES}

Ali, I.K., McKendrick, L., Morley, S.J., and Jackson, R.J. 2001. Activity of the hepatitis A virus IRES requires association between the capbinding translation initiation factor (eIF4E) and eIF4G. J. Virol. 75: 7854-7863.

Altmann, M. and Trachsel, H. 1997. Translation initiation factordependent extracts from yeast Saccharomyces cerevisiae. Methods 11: $343-352$.

Altmann, M., Sonenberg, N., and Trachsel, H. 1989. Translation in Saccharomyces cerevisiae: Initiation factor 4E-dependent cell-free system. Mol. Cell. Biol. 9: 4467-4472.

Asano, K., Shalev, A., Phan, L., Nielsen, K., Clayton, J., Valasek, L., Donahue, T.F., and Hinnebusch, A.G. 2001. Multiple roles for the C-terminal domain of eIF5 in translation initiation complex assembly and GTPase activation. EMBO J. 20: 2326-2337.

Ausubel, F.M., Brent, R., Kingston, R.E., Moore, D.D., Smith, J.A., Seidman, J.D., and Struhl, K. 1989. Current protocols in molecular biology. Greene and Wiley, New York.

Baranick, B.T., Lemp, N.A., Nagashima, J., Hiraoka, K., Kasahara, N., and Logg, C.R. 2008. Splicing mediates the activity of four putative cellular internal ribosome entry sites. Proc. Natl. Acad. Sci. 105: 4733-4738.
Barna, M., Pusic, A., Zollo, O., Costa, M., Kondrashov, N., Rego, E., Rao, P.H., and Ruggero, D. 2008. Suppression of Myc oncogenic activity by ribosomal protein haploinsufficiency. Nature 456: 971-975.

Berlioz, C. and Darlix, J.L. 1995. An internal ribosomal entry mechanism promotes translation of murine leukemia virus gag polyprotein precursors. J. Virol. 69: 2214-2222.

Bieleski, L. and Talbot, S.J. 2001. Kaposi's sarcoma-associated herpesvirus vCyclin open reading frame contains an internal ribosome entry site. J. Virol. 75: 1864-1869.

Boehringer, D., Thermann, R., Ostareck-Lederer, A., Lewis, J.D., and Stark, H. 2005. Structure of the hepatitis C virus IRES bound to the human $80 \mathrm{~S}$ ribosome: remodeling of the HCV IRES. Structure 13: $1695-1706$.

Brasey, A., Lopez-Lastra, M., Ohlmann, T., Beerens, N., Berkhout, B., Darlix, J.L., and Sonenberg, N. 2003. The leader of human immunodeficiency virus type 1 genomic RNA harbors an internal ribosome entry segment that is active during the G2/M phase of the cell cycle. J. Virol. 77: 3939-3949.

Buck, C.B., Shen, X., Egan, M.A., Pierson, T.C., Walker, C.M., and Siliciano, R.F. 2001. The human immunodeficiency virus type 1 gag gene encodes an internal ribosome entry site. J. Virol. 75: 181-191.

Bushman, J.L., Asuru, A.I., Matts, R.L., and Hinnebusch, A.G. 1993. Evidence that GCD6 and GCD7, translational regulators of GCN4, are subunits of the guanine nucleotide exchange factor for eIF-2 in Saccharomyces cerevisiae. Mol. Cell. Biol. 13: 1920-1932.

Butt, T.R., Sternberg, E.J., Gorman, J.A., Clark, P., Hamer, D., Rosenberg, M., and Crooke, S.T. 1984. Copper metallothionein of yeast, structure of the gene, and regulation of expression. Proc. Natl. Acad. Sci. 81: 3332-3336.

Cherry, S., Doukas, T., Armknecht, S., Whelan, S., Wang, H., Sarnow, P., and Perrimon, N. 2005. Genome-wide RNAi screen reveals a specific sensitivity of IRES-containing RNA viruses to host translation inhibition. Genes \& Dev. 19: 445-452.

Choi, S.K., Olsen, D.S., Roll-Mecak, A., Martung, A., Remo, K.L., Burley, S.K., Hinnebusch, A.G., and Dever, T.E. 2000. Physical and functional interaction between the eukaryotic orthologs of prokaryotic translation initiation factors IF1 and IF2. Mol. Cell. Biol. 20: 7183-7191.

Cornelis, S., Bruynooghe, Y., Denecker, G., Van Huffel, S., Tinton, S., and Beyaert, R. 2000. Identification and characterization of a novel cell cycle-regulated internal ribosome entry site. Mol. Cell 5: 597605.

Costantino, D. and Kieft, J.S. 2005. A preformed compact ribosomebinding domain in the cricket paralysis-like virus IRES RNAs. RNA 11: 332-343.

Costantino, D.A., Pfingsten, J.S., Rambo, R.P., and Kieft, J.S. 2008. tRNA-mRNA mimicry drives translation initiation from a viral IRES. Nat. Struct. Mol. Biol. 15: 57-64.

Fernandez, J., Yaman, I., Mishra, R., Merrick, W.C., Snider, M.D., Lamers, W.H., and Hatzoglou, M. 2001. Internal ribosome entry site-mediated translation of a mammalian mRNA is regulated by amino acid availability. J. Biol. Chem. 276: 12285-12291.

Fringer, J.M., Acker, M.G., Fekete, C.A., Lorsch, J.R., and Dever, T.E. 2007. Coupled release of eukaryotic translation initiation factors $5 \mathrm{~B}$ and $1 \mathrm{~A}$ from $80 \mathrm{~S}$ ribosomes following subunit joining. Mol. Cell. Biol. 27: 2384-2397.

Gilbert, W.V., Zhou, K., Butler, T.K., and Doudna, J.A. 2007. Capindependent translation is required for starvation-induced differentiation in yeast. Science 317: 1224-1227.

Goyer, C., Altmann, M., Lee, H.S., Blanc, A., Deshmukh, M., Woolford Jr., J.L., Trachsel, H., and Sonenberg, N. 1993. TIF4631 and TIF4632: two yeast genes encoding the high-molecularweight subunits of the cap-binding protein complex (eukaryotic initiation factor $4 \mathrm{~F}$ ) contain an RNA recognition motif-like sequence and carry out an essential function. Mol. Cell. Biol. 13: 4860-4874.

Grundhoff, A. and Ganem, D. 2001. Mechanisms governing expression of the v-FLIP gene of Kaposi's sarcoma-associated herpesvirus. J. Virol. 75: 1857-1863. 
Hellen, C.U. and Sarnow, P. 2001. Internal ribosome entry sites in eukaryotic mRNA molecules. Genes \& Dev. 15: 1593-1612.

Herbreteau, C.H., Weill, L., Decimo, D., Prevot, D., Darlix, J.L., Sargueil, B., and Ohlmann, T. 2005. HIV-2 genomic RNA contains a novel type of IRES located downstream of its initiation codon. Nat. Struct. Mol. Biol. 12: 1001-1007.

Hernandez, G. 2008. Was the initiation of translation in early eukaryotes IRES-driven? Trends Biochem. Sci. 33: 58-64.

Hershey, J.W.B. and Merrick, W.C. 2000. Pathway and mechanism of initiation of protein synthesis. In Translational control (eds. N. Sonenberg, et al.), pp. 185-243. Cold Spring Harbor Press, Cold Spring Harbor, NY.

Hinnebusch, A.G., Dever, T.E., and Asano, K. 2007. Mechanism of translation initiation in the yeast Saccharomyces cerevisiae. In Translational control in biology and medicine (eds. N. Sonenberg, et al.), pp. 225-268. Cold Spring Harbor Laboratory Press, Cold Spring Harbor, NY.

Jackson, R.J. 2005. Alternative mechanisms of initiating translation of mammalian mRNAs. Biochem. Soc. Trans. 33: 1231-1241.

Jan, E. and Sarnow, P. 2002. Factorless ribosome assembly on the internal ribosome entry site of cricket paralysis virus. J. Mol. Biol. 324: 889-902.

Jan, E., Kinzy, T.G., and Sarnow, P. 2003. Divergent tRNA-like element supports initiation, elongation, and termination of protein biosynthesis. Proc. Natl. Acad. Sci. 100: 15410-15415.

Jang, S.K., Krausslich, H.G., Nicklin, M.J., Duke, G.M., Palmenberg, A.C., and Wimmer, E. 1988. A segment of the $5^{\prime}$ nontranslated region of encephalomyocarditis virus RNA directs internal entry of ribosomes during in vitro translation. J. Virol. 62: 2636-2643.

Jivotovskaya, A.V., Valasek, L., Hinnebusch, A.G., and Nielsen, K.H. 2006. Eukaryotic translation initiation factor 3 (eIF3) and eIF2 can promote mRNA binding to $40 \mathrm{~S}$ subunits independently of eIF4G in yeast. Mol. Cell. Biol. 26: 1355-1372.

Kanamori, Y. and Nakashima, N. 2001. A tertiary structure model of the internal ribosome entry site (IRES) for methionine-independent initiation of translation. RNA 7: 266-274.

Kolupaeva, V.G., Unbehaun, A., Lomakin, I.B., Hellen, C.U., and Pestova, T.V. 2005. Binding of eukaryotic initiation factor 3 to ribosomal 40S subunits and its role in ribosomal dissociation and anti-association. RNA 11: 470-486.

Komar, A.A., Lesnik, T., Cullin, C., Merrick, W.C., Trachsel, H., and Altmann, M. 2003. Internal initiation drives the synthesis of Ure2 protein lacking the prion domain and affects [URE3] propagation in yeast cells. EMBO J. 22: 1199-1209.

Lancaster, A.M., Jan, E., and Sarnow, P. 2006. Initiation factorindependent translation mediated by the hepatitis $\mathrm{C}$ virus internal ribosome entry site. RNA 12: 894-902.

Lee, J.H., Pestova, T.V., Shin, B.S., Cao, C., Choi, S.K., and Dever, T.E. 2002. Initiation factor eIF5B catalyzes second GTP-dependent step in eukaryotic translation initiation. Proc. Natl. Acad. Sci. 99: 16689-16694.

Low, W., Harries, M., Ye, H., Du, M.Q., Boshoff, C., and Collins, M. 2001. Internal ribosome entry site regulates translation of Kaposi's sarcoma-associated herpesvirus FLICE inhibitory protein. J. Virol. 75: 2938-2945.

Majumdar, R., Bandyopadhyay, A., and Maitra, U. 2003. Mammalian translation initiation factor eIF1 functions with eIF1A and eIF3 in the formation of a stable $40 \mathrm{~S}$ preinitiation complex. J. Biol. Chem. 278: 6580-6587.

Marton, M.J., Crouch, D., and Hinnebusch, A.G. 1993. GCN1, a translational activator of GCN4 in Saccharomyces cerevisiae, is required for phosphorylation of eukaryotic translation initiation factor 2 by protein kinase GCN2. Mol. Cell. Biol. 13: 3541-3556.

Mascorro-Gallardo, J.O., Covarrubias, A.A., and Gaxiola, R. 1996. Construction of a CUP1 promoter-based vector to modulate gene expression in Saccharomyces cerevisiae. Gene 172: 169-170.

Nicholson, M.G., Rue, S.M., Clements, J.E., and Barber, S.A. 2006. An internal ribosome entry site promotes translation of a novel SIV Pr55(Gag) isoform. Virology 349: 325-334.
Nielsen, K.H., Szamecz, B., Valasek, L., Jivotovskaya, A., Shin, B.S., and Hinnebusch, A.G. 2004. Functions of eIF3 downstream of $48 \mathrm{~S}$ assembly impact AUG recognition and GCN4 translational control. EMBO J. 23: 1166-1177.

Paz, I., Abramovitz, L., and Choder, M. 1999. Starved Saccharomyces cerevisiae cells have the capacity to support internal initiation of translation. J. Biol. Chem. 274: 21741-21745.

Pelletier, J. and Sonenberg, N. 1988. Internal initiation of translation of eukaryotic mRNA directed by a sequence derived from poliovirus RNA. Nature 334: 320-325.

Pestova, T.V. and Hellen, C.U. 2003. Translation elongation after assembly of ribosomes on the cricket paralysis virus internal ribosomal entry site without initiation factors or initiator tRNA. Genes \& Dev. 17: 181-186.

Pestova, T.V., Lomakin, I.B., Lee, J.H., Choi, S.K., Dever, T.E., and Hellen, C.U. 2000. The joining of ribosomal subunits in eukaryotes requires eIF5B. Nature 403: 332-335.

Pestova, T.V., Lomakin, I.B., and Hellen, C.U. 2004. Position of the CrPV IRES on the $40 S$ subunit and factor dependence of IRES/80S ribosome assembly. EMBO Rep. 5: 906-913.

Pfingsten, J.S. and Kieft, J.S. 2008. RNA structure-based ribosome recruitment: lessons from the Dicistroviridae intergenic region IRESes. RNA 14: 1255-1263.

Pfingsten, J.S., Costantino, D.A., and Kieft, J.S. 2006. Structural basis for ribosome recruitment and manipulation by a viral IRES RNA. Science 314: 1450-1454.

Phan, L., Zhang, X., Asano, K., Anderson, J., Vornlocher, H.P., Greenberg, J.R., Qin, J., and Hinnebusch, A.G. 1998. Identification of a translation initiation factor 3 (eIF3) core complex, conserved in yeast and mammals, that interacts with eIF5. Mol. Cell. Biol. 18: 4935-4946.

Phan, L., Schoenfeld, L.W., Valasek, L., Nielsen, K.H., and Hinnebusch, A.G. 2001. A subcomplex of three eIF3 subunits binds eIF1 and eIF5 and stimulates ribosome binding of mRNA and tRNA $\mathrm{A}_{\mathrm{i}}^{\mathrm{Met}}$. EMBO J. 20: 2954-2965.

Pyronnet, S., Pradayrol, L., and Sonenberg, N. 2000. A cell cycledependent internal ribosome entry site. Mol. Cell 5: 607-616.

Rotenberg, M.O., Moritz, M., and Woolford Jr., J.L. 1988. Depletion of Saccharomyces cerevisiae ribosomal protein L16 causes a decrease in $60 \mathrm{~S}$ ribosomal subunits and formation of half-mer polyribosomes. Genes \& Dev. 2: 160-172.

Sangthong, P., Hughes, J., and McCarthy, J.E. 2007. Distributed control for recruitment, scanning and subunit joining steps of translation initiation. Nucleic Acids Res. 35: 3573-3580.

Schuler, M., Connell, S.R., Lescoute, A., Giesebrecht, J., Dabrowski, M., Schroeer, B., Mielke, T., Penczek, P.A., Westhof, E., and Spahn, C.M. 2006. Structure of the ribosome-bound cricket paralysis virus IRES RNA. Nat. Struct. Mol. Biol. 13: 1092-1096.

Spahn, C.M., Kieft, J.S., Grassucci, R.A., Penczek, P.A., Zhou, K., Doudna, J.A., and Frank, J. 2001. Hepatitis C virus IRES RNAinduced changes in the conformation of the 40s ribosomal subunit. Science 291: 1959-1962.

Spahn, C.M., Jan, E., Mulder, A., Grassucci, R.A., Sarnow, P., and Frank, J. 2004. Cryo-EM visualization of a viral internal ribosome entry site bound to human ribosomes: The IRES functions as an RNA-based translation factor. Cell 118: 465-475.

Stoneley, M. and Willis, A.E. 2004. Cellular internal ribosome entry segments: Structures, trans-acting factors and regulation of gene expression. Oncogene 23: 3200-3207.

Sung, D. and Kang, H. 1998. The N-terminal amino acid sequences of the firefly luciferase are important for the stability of the enzyme. Photochem. Photobiol. 68: 749-753.

Terenin, I.M., Dmitriev, S.E., Andreev, D.E., and Shatsky, I.N. 2008. Eukaryotic translation initiation machinery can operate in a bacteriallike mode without eIF2. Nat. Struct. Mol. Biol. 15: 836-841.

Thompson, S.R., Gulyas, K.D., and Sarnow, P. 2001. Internal initiation in Saccharomyces cerevisiae mediated by an initiator tRNA/eIF2-independent internal ribosome entry site element. Proc. Natl. Acad. Sci. 98: 12972-12977. 
Vagner, S., Waysbort, A., Marenda, M., Gensac, M.C., Amalric, F., and Prats, A.C. 1995. Alternative translation initiation of the Moloney murine leukemia virus mRNA controlled by internal ribosome entry involving the p57/PTB splicing factor. J. Biol. Chem. 270: 20376-20383.

Wilson, J.E., Pestova, T.V., Hellen, C.U., and Sarnow, P. 2000a. Initiation of protein synthesis from the A site of the ribosome. Cell 102: 511-520.

Wilson, J.E., Powell, M.J., Hoover, S.E., and Sarnow, P. 2000 b. Naturally occurring dicistronic cricket paralysis virus RNA is regulated by two internal ribosome entry sites. Mol. Cell. Biol. 20: 4990-4999.

Winzeler, E.A., Shoemaker, D.D., Astromoff, A., Liang, H., Anderson, K., Andre, B., Bangham, R., Benito, R., Boeke, J.D., Bussey, H., et al. 1999. Functional characterization of the $S$. cerevisiae genome by gene deletion and parallel analysis. Science 285: 901-906.

Zhou, W., Edelman, G.M., and Mauro, V.P. 2001. Transcript leader regions of two Saccharomyces cerevisiae mRNAs contain internal ribosome entry sites that function in living cells. Proc. Natl. Acad. Sci. 98: 1531-1536. 

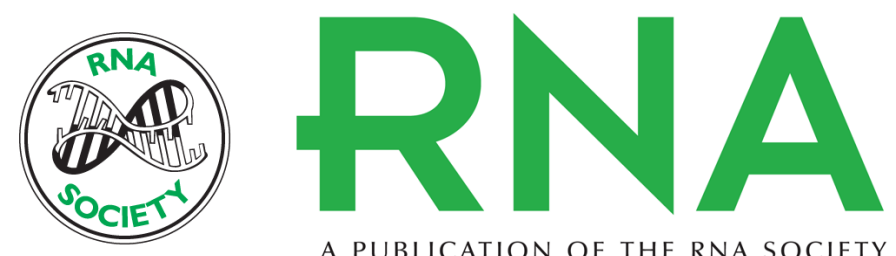

A PUBLICATION OF THE RNA SOCIETY

\section{Translation initiation factors are not required for Dicistroviridae IRES function in vivo}

Nilsa Deniz, Erik M. Lenarcic, Dori M. Landry, et al.

RNA 2009 15: $932-946$ originally published online March 19, 2009

Access the most recent version at doi:10.1261/rna.1315109

\section{References This article cites 66 articles, 45 of which can be accessed free at: http://rnajournal.cshlp.org/content/15/5/932.full.html\#ref-list-1}

\section{License}
Email Alerting Receive free email alerts when new articles cite this article - sign up in the box at the Service top right corner of the article or click here.

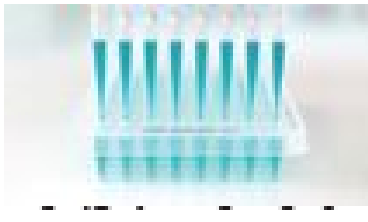

\section{Providing Precise Solutions for} your research.

To subscribe to $R N A$ go to:

http://rnajournal.cshlp.org/subscriptions 